\title{
Work-Energy Principle Based Characteristic Mode Theory for Wireless Power Transfer Systems
}

\author{
Ren-Zun Lian, Ming-Yao Xia, Senior Member, IEEE, and Xing-Yue Guo, Student Member, IEEE
}

\begin{abstract}
Work-energy principle (WEP) governing wireless power transfer (WPT) process is derived. Driving power as the source to sustain a steady WPT is obtained. Transferring coefficient (TC) used to quantify power transfer efficiency is introduced.

WEP gives a clear physical picture to WPT process. The physical picture reveals the essential difference between transferring problem and scattering problem. The essential difference exposes the fact that the conventional characteristic mode theory (CMT) for scattering systems cannot be directly applied to transferring systems.

Under WEP framework, this paper establishes a CMT for transferring systems. By orthogonalizing driving power operator (DPO), the CMT can construct a set of energy-decoupled characteristic modes (CMs) for any pre-selected objective transferring system. It is proved that the obtained $\mathrm{CM}$ set contains the optimally transferring mode, which can maximize TC.

Employing the WEP-based CMT for transferring systems, this paper does the modal analysis for some typical two-coil transferring systems, and introduces the concepts of co-resonance and ci-resonance, and reveals some important differences and connections "between transferring problem and scattering problem", "between co-resonance phenomenon of transferring systems and external resonance phenomenon of scattering systems", and "between so-called magnetic resonance and classical electric-magnetic resonance".
\end{abstract}

Index Terms-Characteristic mode (CM), driving power operator (DPO), transferring coefficient (TC), wireless power transfer (WPT), work-energy principle (WEP).

\section{INTRODUCTION}

$\mathrm{W}$ IRELESS power transfer (WPT) system is a kind of electromagnetic (EM) device designed for wirelessly transferring EM power in an efficiency as high as possible and a distance as long as possible. The earliest researches on WPT can be dated back to the pioneers Hutin and Leblanc [1] and Tesla [2]-[4] etc., and more history on WPT could be found in [5]. According to the difference of working mechanism, WPT systems can be categorized into far-field/radiative WPT systems and near-field/nonradiative WPT systems [6]. The com-

Manuscript with identifier [AP2101-0035] was submitted to IEEE-TAP on January 6, 2021. (Corresponding authors: Ren-Zun Lian; Ming-Yao Xia.)

R. Z. Lian, M. Y. Xia and X. Y. Guo are with the Department of Electronics, School of Electronics Engineering and Computer Science, Peking University, Beijing 100871, China. (e-mail: rzlian@vip.163.com; myxia@pku.edu.cn; gxy4010@126.com)

Color versions of one or more of the figures in this paper are available online at http://ieeexplore.ieee.org.

Digital Object Identifier: AP2101-0035. monly used far-field WPT systems include microwave [7]-[8] and laser [9]-[10] WPT systems etc. The commonly used near-field WPT systems include inductive [11]-[13], capacitive [14]-[15], conductive [16]-[17], and magnetic resonance [18]-[23] WPT systems etc.

In the various WPT systems, the magnetic-resonance-based systems seem more desired in the applications of medium to high power levels, and more advantageous in the aspects of transferring capability, transferring efficiency, safety, and controllability [22]-[23]. The magnetic resonance WPT systems are focused on by this paper, and the principles and formulations obtained in this paper can be further generalized to the other kinds of WPT systems in the future. For convenience, the magnetic resonance WPT systems are simply called transferring systems in the following discussions.

A classical transferring system is shown in Fig. 1. The transferring system is constituted by two metallic coils transmitting coil $\mathrm{T}$ and receiving coil $\mathrm{R}$. Coil $\mathrm{T}$ is excited by a locally impressed driver (e.g. delta-gap source) used to inject power into coil $\mathrm{T}$. Coil $\mathrm{R}$ is connected to a perfectly matched load (e.g. matched light bulb) used to extract power from coil R. The power is transmitted by coil $\mathrm{T}$, and then wirelessly transferred from coil $\mathrm{T}$ to coil $\mathrm{R}$ by passing through the environment surrounding transferring system, and finally received by coil R.

Inspired by the acoustic resonance between acoustic resonators, Tesla introduced the concept of magnetic resonance to the realm of WPT for the first time, and patented the well-known "Tesla coil" [24]. In 2007, Kurs et al. [18]-[19] employ the magnetic resonance between the coils $\mathrm{T}$ and $\mathrm{R}$ shown in Fig. 1 to realize fully lighting up a 60-W light bulb from distances more than $2 \mathrm{~m}$ away. The time-average magnetic energy density distribution of the desired transferring mode is visualized in Fig. 2. Evidently, the mode shown in Fig. 2 indeed has ability to efficiently transfer power from coil $\mathrm{T}$ to coil $\mathrm{R}$ wirelessly. In recent years, the magnetic-resonance-based WPT technology has been widely applied in the realms of wirelessly charging consumer electronic products [25], electric vehicles (EV) [26]-[27], biomedical implants [28]-[30], underwater devices [31]-[32], Internet of things (IoT) [33]-[34], and industrial robots [35]-[36] etc.

Obviously, a systematical modal analysis method will significantly facilitate the theoretical analysis and engineering design for transferring systems. In fact, there have existed some different kinds of modal analysis methods for transferring systems, such as coupled-mode theory [18]-[19], [37], classical circuit theory [38]-[40], and some other theories [41]-[42], and 
all of them are based on circuit models. However, the circuit-model-based modal analysis methods need to use some circuit-based concepts, such as scalar voltage, scalar current, self-inductance, mutual inductance, and capacitance etc., so they are some approximate but not rigorous methods. In addition, the employment for scalar voltage and scalar current implies that the circuit-model-based modal analysis methods are only applicable to the transferring systems working at low frequency and with simple geometrical structures (such as rectangular coils and circular coils [18]-[23] etc.). Thus, it is one of important challenges in the realm of magnetic-resonance-based WPT how to develop a rigorous, frequency-independent, and geometry-independent modal analysis method for transferring systems.

The conventional characteristic mode theory (CMT) [43]-[48] is just a rigorous, frequency-independent, and geometry-independent modal analysis method. However, the conventional CMT is a method for scattering systems as revealed by its physical picture [47]-[48], but cannot be directly applied to transferring systems (due to the different working mechanisms of scattering and transferring systems, for details please see Sec. II) as exhibited in the following example. A direct application of the conventional CMT to the transferring system shown in Fig. 1 outputs some CMs, and the resonant CM has the time-average magnetic energy density distribution shown in Fig. 3. Evidently, the resonant CM shown in Fig. 3 doesn't realize the most efficient WPT from coil $\mathrm{T}$ to coil R like Fig. 2.

The one shown in Fig. 1 is a simplest and most classical metallic transferring system. Using it as a typical example, this paper is devoted to generalizing the conventional CMT for scattering systems (simply called scattering CMT) to a novel CMT for transferring systems (simply called transferring CMT), and this paper is organized as follows: Sec. II discusses the physical principle governing the working mechanism of transferring problem; Sec. III provides the mathematical formulas used to establish the transferring CMT; Sec. IV employs the CMT to do some modal analysis for the classical transferring system shown in Fig. 2 to exhibit the validity of the theory; Sec. V applies the transferring CMT to some typical variants of the classical transferring system for exhibiting the wide applicable range of the theory; Sec. VI concludes this paper; Apps. A-D provide some detailed formulations related to this paper.

In what follows, the $e^{j \omega t}$ convention and inner product $<\boldsymbol{f}, \boldsymbol{g}>_{\Omega}=\int_{\Omega} \boldsymbol{f}^{\dagger} \cdot \boldsymbol{g} d \Omega$ are used throughout, where superscript " $\uparrow$ " is the conjugate transpose operation for a scalar/vector/matrix. The environment surrounding transferring system is free space, and its permeability and permittivity are denoted as $\mu_{0}$ and $\varepsilon_{0}$ respectively. Time-domain and frequency-domain powers are denoted as $\mathcal{P}$ and $P$ respectively; time-domain and frequency-domain currents and fields are denoted as $(\boldsymbol{J}, \mathcal{F})$ and $(\boldsymbol{J}, \boldsymbol{F})$ respectively; frequency-domain power quadratic matrix and current expansion coefficient vector are denoted as $\mathrm{P}$ and $\mathrm{J}$ respectively. In addition, for the linear quantities (e.g. electric field intensity), we have that $\mathcal{E}=\operatorname{Re}\left\{\boldsymbol{E} e^{j \omega t}\right\}$; for the power-type quadratic quantity, we have that $\operatorname{Re}\left\{(1 / 2) \boldsymbol{J}^{\dagger} \cdot \boldsymbol{E}\right\}=(1 / T) \int_{0}^{\dagger} \boldsymbol{J} \cdot \mathcal{E} d t$, where $T$ is the time period of the time-harmonic EM field.

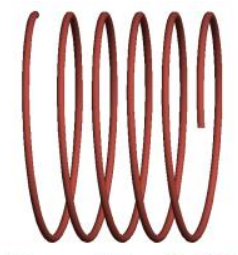

Transmitting Coil T

Fig. 1. A classical magnetic resonance WPT system constituted by a metallic transmitting coil $\mathrm{T}$ and a metallic receiving coil R. For the one reported in [18], the coils have the same radius $30 \mathrm{~cm}$, height $20 \mathrm{~cm}$, and turns 5.25 .

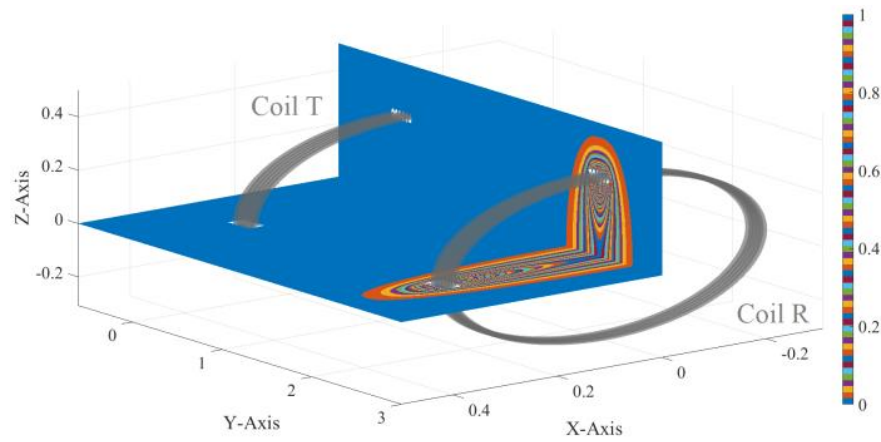

Fig. 2. Time-average magnetic energy density distribution of the desired transferring mode reported in [18]. The energy is almost completely transferred from coil $\mathrm{T}$ to coil $\mathrm{R}$.

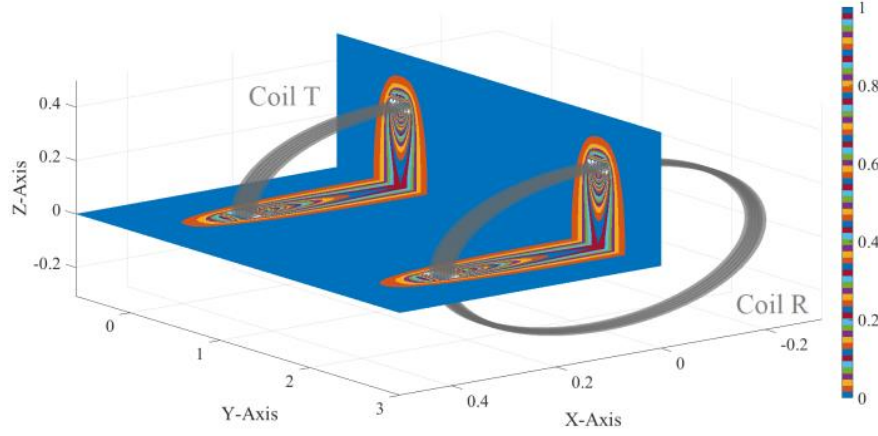

Fig. 3. Time-average magnetic energy density distribution of the resonant CM calculated from the conventional CMT for scattering systems [45]-[47]. The energy oscillates locally, but it is not transferred from coil T to coil R.

\section{PhysicAl PRINCIPLES}

For the transferring system shown in Fig. 1, a typical locally impressed driver is delta-gap source, and the source provides a voltage driving to coil $\mathrm{T}$. The voltage driving has a field effect, i.e., the voltage driving can be equivalently viewed as a field driving. The voltage driving acts on coil T only (but doesn't act on coil $\mathrm{R}$ and load), so the equivalent field driving also acts on coil T only (but doesn't act on coil $\mathrm{R}$ and load), i.e., the driving field is localized/restricted in the region occupied by coil $\mathrm{T}$, and this is just the reason to call it locally impressed driver.

The action of the driving field on coil $\mathrm{T}$ will induce a current on coil $\mathrm{T}$, and the current will generate a field on surrounding environment. Similarly, the field generated by coil $\mathrm{T}$ will act on coil R, and the action will lead to an induced current on coil R, and the current will generate a field on surrounding environment. In addition, the fields generated by the coils act on the load as well.

In fact, there also exists a reaction from the field generated by coil $\mathrm{R}$ to the current distributing on coil $\mathrm{T}$, and the reaction 
will affect the current distribution. However, there doesn't exist any reaction from the load to the coils, i.e., the field effect of the load is localized/restricted in the region occupied by the load itself, because the load is supposed as a perfectly matched one in this paper.

Through a complicated process, the above actions and reactions will reach a dynamic equilibrium finally, because the EM problem considered here is time-harmonic.

For the convenience of following discussions, the boundary surfaces of coil T and coil R are denoted as $S_{\mathrm{t}}$ and $S_{\mathrm{r}}$ respectively; the three-dimensional Euclidean space is denoted as $\mathbb{E}^{3}$; the boundary of $\mathbb{E}^{3}$ is denoted as $S_{\infty}$, which is a closed spherical surface with infinite radius. The driving field generated by locally impressed driver is denoted as $\mathcal{F}_{\text {driv }}$. At the state of dynamic equilibrium, the currents distributing on coil $\mathrm{T}$ and coil R are denoted as $\boldsymbol{J}_{\mathrm{t}}$ and $\boldsymbol{J}_{\mathrm{r}}$ respectively. The fields generated by $\boldsymbol{J}_{\mathrm{t}}$ and $\boldsymbol{J}_{\mathrm{r}}$ are denoted as $\mathcal{F}_{\mathrm{t}}$ and $\mathcal{F}_{\mathrm{r}}$ respectively.

The energy conservation law tells us that the above actions and reactions among driver, coil $\mathrm{T}$, coil $\mathrm{R}$, and load will result in a work-energy transformation, and the work-energy transformation can be quantitatively expressed as follows:

$$
\mathcal{P}_{\text {driv }}=\mathcal{P}_{\text {sel }}^{\mathrm{tt}}+\mathcal{P}_{\text {tra }}
$$

called time-domain work-energy principle (WEP), where $\mathcal{P}_{\text {driv }}=\left\langle\boldsymbol{J}_{\mathrm{t}}, \mathcal{E}_{\text {driv }}\right\rangle_{S_{\mathrm{t}}}$ and $\left.\mathcal{P}_{\text {sel }}^{\mathrm{tt}}=-<\boldsymbol{J}_{\mathrm{t}}, \mathcal{E}_{\mathrm{t}}\right\rangle_{S_{\mathrm{t}}}$ and $\left.\mathcal{P}_{\text {tra }}=-<\boldsymbol{J}_{\mathrm{t}}, \mathcal{E}_{\mathrm{r}}\right\rangle_{S_{\mathrm{t}}}$. A rigorous mathematical derivation for WEP (1) and a more detailed decomposition for $\mathcal{P}_{\text {driv }}$ are provided in App. A.

Similar to the scattering problem discussed in [47]-[48], the current-field interaction $\left\langle\boldsymbol{J}_{\mathrm{t}}, \boldsymbol{\mathcal { E }}_{\text {driv }}\right\rangle_{S_{\mathrm{t}}}$ is the source for driving a steady work-energy transformation, so $\mathcal{P}_{\text {driv }}$ is called driving power. Based on Maxwell's equations, the current-field interaction $\left.-<\boldsymbol{J}_{\mathrm{t}}, \mathcal{E}_{\mathrm{t}}\right\rangle_{S_{\mathrm{t}}}$ can be alternatively written as follows:

$$
\begin{aligned}
-\left\langle\boldsymbol{J}_{\mathrm{t}}, \mathcal{E}_{\mathrm{t}}\right\rangle_{S_{\mathrm{t}}}= & \oiint_{S_{\infty}}\left(\mathcal{E}_{\mathrm{t}} \times \mathcal{H}_{\mathrm{t}}\right) \cdot d \boldsymbol{S} \\
& +\frac{d}{d t}\left[(1 / 2)\left\langle\mathcal{H}_{\mathrm{t}}, \mathcal{B}_{\mathrm{t}}\right\rangle_{\mathbb{E}^{3}}+(1 / 2)\left\langle\mathcal{D}_{\mathrm{t}}, \mathcal{E}_{\mathrm{t}}\right\rangle_{\mathbb{E}^{3}}\right]
\end{aligned}
$$

called Poynting's theorem, where $\mathcal{D}_{\mathrm{t}}=\varepsilon_{0} \mathcal{E}_{\mathrm{t}}$ and $\mathcal{B}_{\mathrm{t}}=\mu_{0} \mathcal{H}_{\mathrm{t}}$. The theorem quantitatively governs the way how $\boldsymbol{J}_{\mathrm{t}}$ provides EM power to $\mathcal{F}_{\mathrm{t}}$, so $\mathcal{P}_{\text {sel }}^{\text {tt }}$ is called self-power of coil T. It has been explained previously that coil $\mathrm{R}$ is driven by coil $\mathrm{T}$, and it will be further proven in Sec. III that $J_{\mathrm{r}}$ is uniquely determined by $\boldsymbol{J}_{\mathrm{t}}$, so $\boldsymbol{J}_{\mathrm{t}}$ can be viewed as the source for providing power to $\mathcal{F}_{\mathrm{r}}$ and the power can be expressed as current-field interaction $-<\boldsymbol{J}_{\mathrm{t}}, \boldsymbol{\mathcal { E }}_{\mathrm{r}}>_{S_{\mathrm{t}}}$ (which is similar to the current-field interaction $-<\boldsymbol{J}_{\mathrm{t}}, \mathcal{E}_{\mathrm{t}}>_{S_{\mathrm{t}}}$ of the coil $\mathrm{T}$ itself), and then $\mathcal{P}_{\text {tra }}$ is called transferred power from coil $\mathrm{T}$ to coil $\mathrm{R}$.

The above these clearly reveal the physical picture of WPT process: driver drives coil $\mathrm{T}$, and the driving power $\mathcal{P}_{\text {driv }}$ is transformed into two parts $\mathcal{P}_{\text {sel }}^{\mathrm{tt}}$ and $\mathcal{P}_{\text {tra }}$ by the transferring system, where $\mathcal{P}_{\text {sel }}^{\text {tt }}$ is dissipated by coil T and $\mathcal{P}_{\text {tra }}$ is wirelessly transferred from coil $\mathrm{T}$ to coil $\mathrm{R}$. The physical picture clearly reveals the fact that the working mechanism of transferring systems is different from the working mechanism of scattering systems (for details of the latter please see [47] and [48]). This is just the reason why the scattering CMT [43]-[48] cannot be directly applied to transferring systems.

For the WPT application, the transferred power $\mathcal{P}_{\text {tra }}$ is desired, and the dissipated power $\mathcal{P}_{\text {sel }}^{\mathrm{tt}}$ is unwanted and expected to be as small as possible, so we introduce a novel concept of transferring coefficient (TC) as follows:

$$
\mathrm{TC}=\frac{(1 / T) \int_{0}^{T} \mathcal{P}_{\text {tra }} d t}{(1 / T) \int_{0}^{T} \mathcal{P}_{\text {driv }} d t}
$$

to quantify the transferring efficiency of transferring system. From a relatively mathematical viewpoint, the central aim of designing transferring system is to search for a physically realizable working mode (or working state) such that TC is maximized. In this paper, the central aim is realized by applying a CMT-based modal analysis to the transferring system.

In the following Sec. III, we provide the mathematical formulations used to establish the WEP-based CMT (WEP-CMT) for transferring systems. Because there exist relations $(1 / T) \int_{0}^{T} \mathcal{P}_{\text {tra }} d t=\operatorname{Re}\left\{P_{\text {tra }}\right\}$ and $(1 / T) \int_{0}^{T} \mathcal{P}_{\text {driv }} d t=\operatorname{Re}\left\{P_{\text {driv }}\right\}$, then the following Sec. III is discussed in frequency domain.

\section{MATHEMATICAL FoRMULATIONS}

The EM field boundary condition on $S_{\mathrm{t}}$ implies the relation that $\left[\boldsymbol{E}_{\mathrm{driv}}\right]_{\mathrm{tan}}=\left[-\boldsymbol{E}_{\mathrm{t}}-\boldsymbol{E}_{\mathrm{r}}\right]_{\mathrm{tan}}$ on $\boldsymbol{S}_{\mathrm{t}}$, where subscript "tan" represents that the relation is satisfied by the tangential component. Electric fields $\boldsymbol{E}_{\mathrm{t}}$ and $\boldsymbol{E}_{\mathrm{r}}$ have operator expressions $\boldsymbol{E}_{\mathrm{t}}=-j \omega \mu_{0} \mathcal{L}_{0}\left(\boldsymbol{J}_{\mathrm{t}}\right)$ and $\boldsymbol{E}_{\mathrm{r}}=-j \omega \mu_{0} \mathcal{L}_{0}\left(\boldsymbol{J}_{\mathrm{r}}\right)$ respectively, in which $\mathcal{L}_{0}\left(\boldsymbol{J}_{\mathrm{t} / \mathrm{r}}\right)=\left[1+\left(1 / k_{0}^{2}\right) \nabla \nabla \cdot\right] \int_{0} G_{0}\left(\boldsymbol{r}, \boldsymbol{r}^{\prime}\right) \boldsymbol{J}_{\mathrm{t} / \mathrm{r}}\left(\boldsymbol{r}^{\prime}\right) d \Omega^{\prime} \quad$ where $G_{0}\left(\boldsymbol{r}, \boldsymbol{r}^{\prime}\right)=e^{-j k_{0}\left|r-r^{\prime}\right|} / 4 \pi\left|\boldsymbol{r}-\boldsymbol{r}^{\prime}\right|$. Based on the above these, frequency-domain driving power $P_{\text {driv }}$ can be expressed as the following operator form

$$
P_{\text {driv }}=-(1 / 2)\left\langle\boldsymbol{J}_{\mathrm{t}},-j \omega \mu_{0} \mathcal{L}_{0}\left(\boldsymbol{J}_{\mathrm{t}}+\boldsymbol{J}_{\mathrm{r}}\right)\right\rangle_{S_{\mathrm{t}}}
$$

called frequency-domain driving power operator (DPO).

In fact, the physically realizable $\boldsymbol{J}_{\mathrm{t}}$ and $\boldsymbol{J}_{\mathrm{r}}$ are not independent of each other, because they must satisfy the following electric field integral equation

$$
\left[-j \omega \mu_{0} \mathcal{L}_{0}\left(\boldsymbol{J}_{\mathrm{t}}\right)-j \omega \mu_{0} \mathcal{L}_{0}\left(\boldsymbol{J}_{\mathrm{r}}\right)\right]_{\tan }=0 \text { on } S_{\mathrm{r}}
$$

due to the homogeneous tangential electric field boundary condition $\left[\boldsymbol{E}_{\mathrm{t}}+\boldsymbol{E}_{\mathrm{r}}\right]_{\mathrm{tan}}=0$ on $S_{\mathrm{r}}$. Applying the method of moments (MoM) to (5), the integral equation is immediately discretized into a matrix equation. By solving the matrix equation, the following transformation

$$
\mathrm{J}_{\mathrm{r}}=\mathrm{T} \cdot \mathrm{J}_{\mathrm{t}}
$$

can be obtained, where $\mathrm{J}_{\mathrm{t}}$ and $\mathrm{J}_{\mathrm{r}}$ are the column vectors constituted by the expansion coefficients of $\boldsymbol{J}_{\mathrm{t}}$ and $\boldsymbol{J}_{\mathrm{r}}$ respectively. The detailed mathematical process for deriving (6) from 
(5) is provided in App. B.

Similarly to discretizing integral equation (5), the DPO (4) can be discretized into its matrix form. Substituting transformation (6) into the matrix form, we immediately have that

$$
P_{\text {driv }}=\mathrm{J}_{\mathrm{t}}^{\dagger} \cdot \mathrm{P}_{\text {driv }} \cdot \mathrm{J}_{\mathrm{t}}
$$

The detailed mathematical formulations for calculating matrix $P_{\text {driv }}$ are listed in App. C. In fact, the above process from (4) to (7) is just the dependent variable elimination (DVE) process used in the WEP-CMT for scattering systems [47]-[48].

WEP-CMT decomposes $\mathrm{P}_{\text {driv }}$ in terms of its positive and negative Hermitian parts as that $\mathrm{P}_{\text {driv }}=\mathrm{P}_{\text {driv }}^{+}+j \mathrm{P}_{\text {driv }}^{-}$(where $\mathrm{P}_{\text {driv }}^{+}=\left(\mathrm{P}_{\text {driv }}+\mathrm{P}_{\text {driv }}^{\dagger}\right) / 2$ and $\left.\mathrm{P}_{\text {driv }}^{-}=\left(\mathrm{P}_{\text {driv }}-\mathrm{P}_{\text {driv }}^{\dagger}\right) / 2 j\right)$, and constructs $\mathrm{CMs}$ by solving the following characteristic equation

$$
\mathrm{P}_{\text {driv }}^{-} \cdot \mathrm{J}_{\mathrm{t}}=\lambda \mathrm{P}_{\text {driv }}^{+} \cdot \mathrm{J}_{\mathrm{t}}
$$

where $\lambda$ and $\mathrm{J}_{\mathrm{t}}$ are the associated characteristic values and characteristic vectors respectively.

Using the above-obtained characteristic vector $\mathrm{J}_{\mathrm{t}}$, the TC of the $\mathrm{CM}$ can be calculated as follows:

$$
\mathrm{TC}=\frac{\mathrm{J}_{\mathrm{t}}^{\dagger} \cdot \mathrm{P}_{\mathrm{tra}}^{+} \cdot \mathrm{J}_{\mathrm{t}}}{\mathrm{J}_{\mathrm{t}}^{\dagger} \cdot \mathrm{P}_{\text {driv }}^{+} \cdot \mathrm{J}_{\mathrm{t}}}
$$

where $\mathrm{P}_{\text {tra }}^{+}$is given in App. C. By comparing the TCs of the $\mathrm{CMs}$, the $\mathrm{CM}$ with the maximal TC can be easily found, and the CM is usually a desired selection for the WPT application.

In the following Sec. IV, we provide a WEP-CMT-based modal analysis for a classical example, which is just the transferring system reported in the seminal paper [18].

\section{NUMERICAL VERIFICATIONS}

The transferring system considered in [18] is constituted by two metallic coils as shown in Fig. 1. The coils have the same radius $30 \mathrm{~cm}$, height $20 \mathrm{~cm}$, and turns 5.25 . The coils are placed coaxially, and their distance is $2 \mathrm{~m}$. The optimally transferring frequency (i.e. the working frequency of the optimally transferring mode) calculated from the coupled-mode theory used in [18] is $10.56 \pm 0.3 \mathrm{MHz}$, and the optimally transferring frequency obtained from the measurement done in [18] is 9.90 MHz. The reason leading to a $5 \%$ discrepancy between the theoretical and measured values was explained in [18].

\section{A. WEP-CMT-Based Modal Analysis}

We use the WEP-CMT given in Secs. II and III to calculate the CMs of the transferring system, and show the TC curves of the first $5 \mathrm{CMs}$ in Fig. 4. Based on the analysis given in Secs. II and III, it is not difficult to conclude that the CM 1 at 10.8988 $\mathrm{MHz}$ (which corresponds to the local maximum of the TC curve) works at the optimally transferring state. The coil current distribution and time-average magnetic energy density distribution of the optimally transferring mode are shown in Fig. 5 and Fig. 6 respectively.

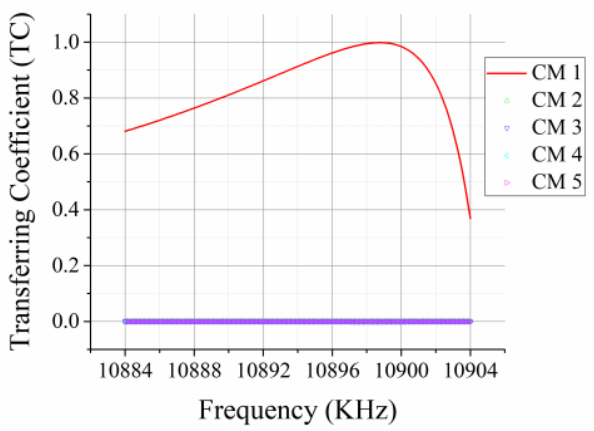

Fig. 4. TC curves of the first 5 low-order CMs calculated from the WEP-CMT established in this paper.
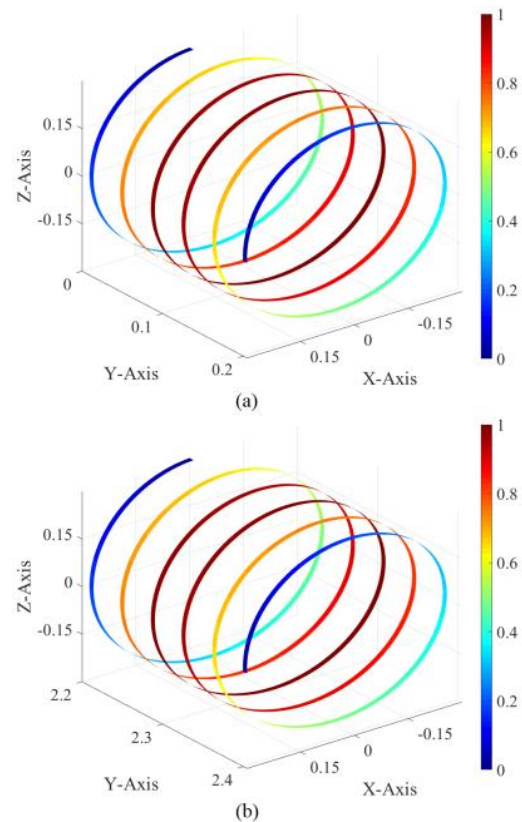

Fig. 5. For the CM 1 working at $10.8988 \mathrm{MHz}$, its current magnitudes distributing on (a) coil $\mathrm{T}$ and (b) coil $\mathrm{R}$.

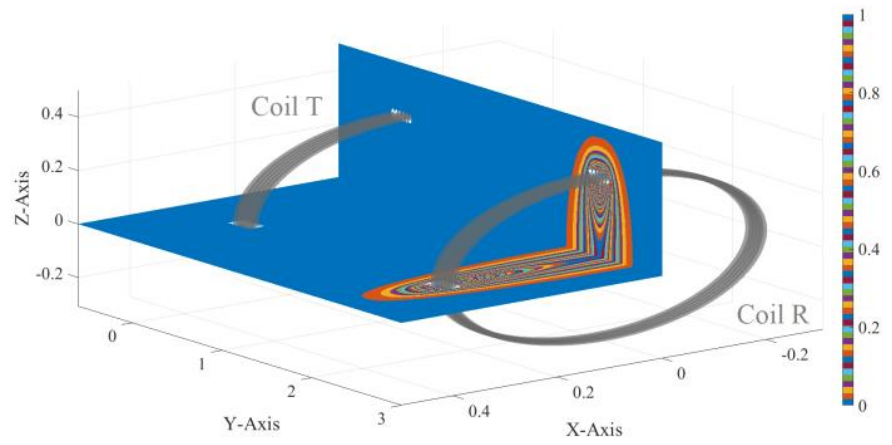

Fig. 6. For the CM 1 working at $10.8988 \mathrm{MHz}$, its time-average magnetic energy density distributing on $\mathrm{xOy}$ and $\mathrm{yOz}$ planes.

Evidently, the CM 1 working at $10.8988 \mathrm{MHz}$ corresponds to a half-wave current distribution for both the coil $\mathrm{T}$ and coil $\mathrm{R}$ as shown in Fig. 5, and it indeed can efficiently transfer EM power from coil $\mathrm{T}$ to coil $\mathrm{R}$ in a wireless manner as shown in Fig. 6.

In addition, the optimally transferring frequencies calculated from the classical coupled-mode theory $(10.56 \pm 0.3 \mathrm{MHz})$ proposed in [18] and the WEP-CMT (10.8988 MHz) used in this paper are consistent with each other. The advantage of WEP-CMT over coupled-mode theory is reflected as follows: 
A1 The WEP-CMT is a field-based modal analysis method, which is directly derived from Maxwell's equations and doesn't use any approximation; the coupled-mode theory is a circuit-model-based modal analysis method, which employs some circuit-model-based approximate quantities (such as scalar voltage, scalar current, effective inductance, and effective capacitance, etc. [18]-[19]).

A2 The WEP-CMT is applicable to the coils working at arbitrary frequency; the coupled-mode theory is only applicable to the coils working at low frequency at which the circuit model exists.

A3 The WEP-CMT is applicable to the coils with arbitrary topological structures; the coupled-mode theory is only applicable to the coils with simple topological structures, such that the coils can support sinusoidal scalar currents.

By employing an alternative field-based modal analysis, the following Sec. IV-B verifies that the CM 1 at $10.8988 \mathrm{MHz}$ is indeed the most efficient mode for WPT, and then exhibits that the optimally transferring mode is indeed included in the CM set constructed by the WEP-CMT.

\section{B. An Alternative Modal Analysis}

Because the matrices $\mathrm{P}_{\text {tra }}^{+}$and $\mathrm{P}_{\text {driv }}^{+}$in (9) are Hermitian, and the matrix $\mathrm{P}_{\text {driv }}^{+}$is positive definite, then the mode maximizing TC can be obtained from solving the following equation [49]

$$
\mathrm{P}_{\text {tra }}^{+} \cdot \mathrm{J}_{\mathrm{t}}=\tau \mathrm{P}_{\text {driv }}^{+} \cdot \mathrm{J}_{\mathrm{t}}
$$

Using the equation, we calculate the optimally transferring mode, and show the associated TC curve in Fig. 7. Obviously, both the obtained optimally transferring frequency and optimally transferring coefficient are consistent with the ones obtained from the WEP-CMT-based modal analysis method.

\section{Concepts of Co-resonance and Ci-resonance}

In this sub-section, we, under the WEP framework, provide some further modal analysis to the CM 1 shown in Fig. 4, and introduce the concepts of co-resonance and ci-resonance.

For the CM 1, its various resistance and reactance curves are shown in Fig. 8. The definitions for the resistances and reactances are given in App. D. From the Figs. 8(a) and 8(b), it is easy to observe the following facts:

F1 At optimally transferring frequency $f_{\text {cor }}=10.8988 \mathrm{MHz}$, $R_{\mathrm{sel}}^{\mathrm{tt}}$ is very small; $R_{\mathrm{mut}}^{\mathrm{tr}}$ is equal to zero; $R_{\mathrm{driv}}, R_{\mathrm{sel}}^{\mathrm{rr}}$, and $R_{\text {tra }}$ achieve their local maximums simultaneously, and the maximal values are the same.

F2 At frequency $f_{\text {cor }}=10.8988 \mathrm{MHz}, X_{\text {sel }}^{\mathrm{tt}}, X_{\text {mut }}^{\mathrm{tr}}$, and $X_{\text {sel }}^{\mathrm{rr}}$ are zero simultaneously, and then $X_{\text {driv }}$ and $X_{\text {tra }}$ are zero automatically because of that $X_{\text {driv }}=X_{\text {sel }}^{\mathrm{tt}}+X_{\text {mut }}^{\mathrm{tr}}+X_{\text {sel }}^{\text {rr }}$ and $X_{\text {tra }}=X_{\text {mut }}^{\mathrm{tr}}+X_{\text {sel }}^{\mathrm{rr}}$.

F3 At frequency $f_{\text {cir }}=10.8873 \mathrm{MHz}, R_{\text {driv }}, R_{\text {sel }}^{\mathrm{tr}}, R_{\text {mut }}^{\mathrm{tr}}, R_{\text {sel }}^{\mathrm{rr}}$, and $R_{\text {tra }}$ are very small.

F4 At frequency $f_{\text {cir }}=10.8873 \mathrm{MHz}$, only $X_{\text {driv }}$ is zero, but the other reactances are not.

The subscripts "cor" and "cir" on $f_{\text {cor }}$ and $f_{\text {cir }}$ are explained as below.

Based on the above observations, it is not difficult to conclude that:

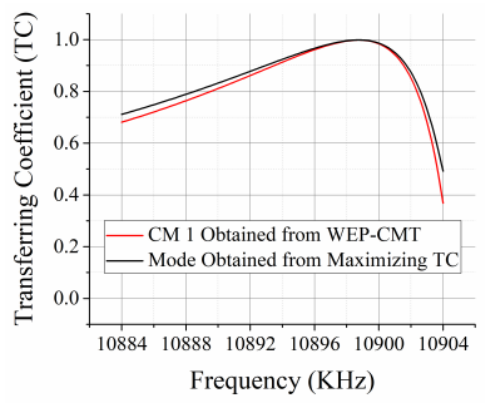

Fig. 7. TC curves of the optimally transferring modes obtained from two somewhat different modal analysis methods provided in this paper.

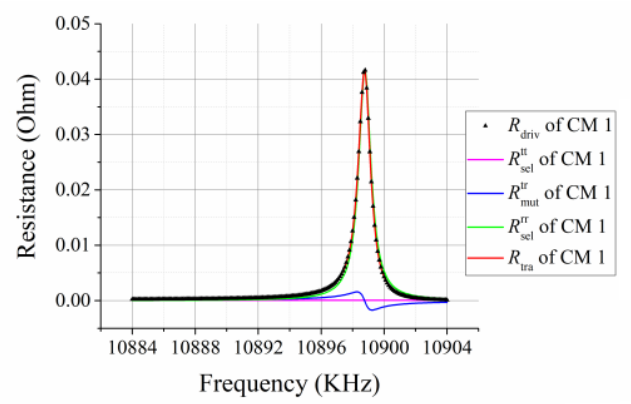

(a)

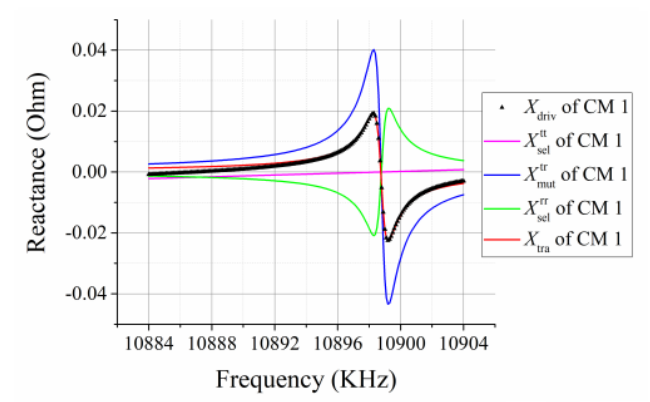

(b)

Fig. 8. For the CM 1, its (a) resistance curves and (b) reactance curves.

C1 At optimally transferring frequency $f_{\text {cor }}$, the driver provides power to coil $\mathrm{T}$ in a very efficient way, and the driving power is almost completely transferred from coil $\mathrm{T}$ to coil R except a very small part dissipated by coil $\mathrm{T}$, and the transferred power is completely converted by coil $\mathrm{R}$ into its self-power but not into any mutual-power.

C2 At optimally transferring frequency $f_{\text {cor }}$, coil $\mathrm{T}$ works at the state of self-resonance (because $X_{\text {sel }}^{\mathrm{tt}}=0$ ), and coil R works at the state of self-resonance as well (because $\left.X_{\text {sel }}^{\mathrm{rr}}=0\right)$, and, at the same time, the two coils also work at the state of mutual-resonance (because $X_{\text {mut }}^{\text {tr }}=0$ ). The working state with $X_{\text {sel }}^{\mathrm{tt}}=X_{\text {mut }}^{\mathrm{tr}}=X_{\text {sel }}^{\mathrm{rr}}=0$ is particularly called co-resonance in this paper, and conventionally called magnetic-resonance in [18]-[23]. In fact, the co-resonance/magnetic-resonance automatically leads to that $X_{\text {tra }}=0$ and $X_{\text {driv }}=0$, because $X_{\text {tra }}=X_{\text {mut }}^{\text {tr }}+X_{\text {sel }}^{\text {rr }}$ and $X_{\text {driv }}=X_{\text {sel }}^{\mathrm{tt}}+X_{\text {mut }}^{\mathrm{tr}}+X_{\text {sel }}^{\mathrm{rr}}$.

C3 The co-resonance/magnetic-resonance satisfies the classical resonance condition that the reactance is zero, i.e., the stored electric energy is equal to the stored magnetic energy. Thus, the co-resonance/magnetic-resonance is a special case of the classical EM-resonance (electric-magnetic resonance). 
C4 At frequency $f_{\text {cir }}$, whole transferring system is resonant (because $X_{\text {driv }}=0$ ), but neither coil $\mathrm{T}$ nor coil $\mathrm{R}$ is resonant (because $X_{\text {sel }}^{\mathrm{tt}}, X_{\text {sel }}^{\mathrm{rr}} \neq 0$ ). The resonance of whole transferring system originates from neutralizing the negative $X_{\text {sel }}^{\mathrm{tt}}+X_{\text {sel }}^{\mathrm{rr}}$ and the positive $X_{\mathrm{mut}}^{\mathrm{tr}}$. To distinguish this kind of resonance from the previous co-resonance, this kind of resonance is particularly called capacitive-inductive-neutralized resonance (simply denoted as ci-resonance) in this paper.

In addition, by observing Fig. 4 and Fig. 8(a), it is easy to find out that the co-resonant mode is more desired than the ci-resonant mode for WPT application, because the former corresponds to the local maximums of TC curve and $R_{\text {tra }}$ curve but the latter doesn't.

\section{TYPICAL EXAMPLES}

To exhibit the values of WEP-CMT in the aspect of analyzing transferring systems and reveal the connections between transferring problem and scattering problem, this section employs WEP-CMT to do some valuable modal analysis for several typical variants of the classical two-coil transferring system considered in Sec. IV.

\section{A. On Transferring Frequency - Part I}

Now, we consider the scattering system only constituted by the coil T considered in Sec. IV, and we calculate its scattering CMs by using the WEP-CMT for scattering systems [45]-[47]. The modal significance (MS) curves associated with the first 5 CMs are shown in Fig. 9. The resonant CM i with the local maximal MS has the same current distribution as the one shown in Fig. 5(a).

Comparing the Fig. 9 with the previous Figs. 4, 7 and 8, it is evident that

C5 The co-resonance frequency (i.e. the optimally transferring frequency) of the two-coil transferring system considered in Sec. IV is equal to the conventional external-resonance frequency of the one-coil scattering system.

This conclusion effectively establishes a connection between the co-resonance-based/magnetic-resonance-based wireless power transferring problem and the conventional scattering problem.

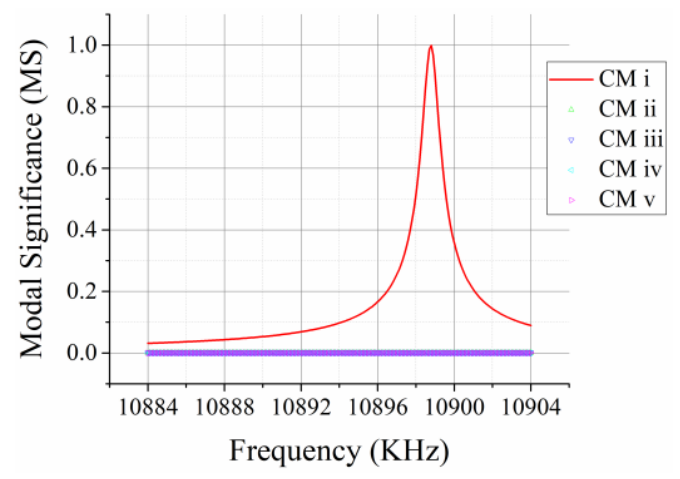

Fig. 9. MS curves of the first 5 CMs of the one-coil scattering system.

\section{B. On Transferring Frequency - Part II}

In fact, the above-mentioned external-resonance frequency is the one-order external-resonance frequency of the one-coil scattering system, and the scattering system has some other higher-order external-resonance frequencies, such as the two-order external-resonance frequency $27.2646 \mathrm{MHz}$ as shown in Figs. 10 and 11.

In the frequency band used in Fig. 10, we calculate the CMs of the two-coil transferring system, and show the TC and reactance curves associated with CM 2 in Fig. 12.

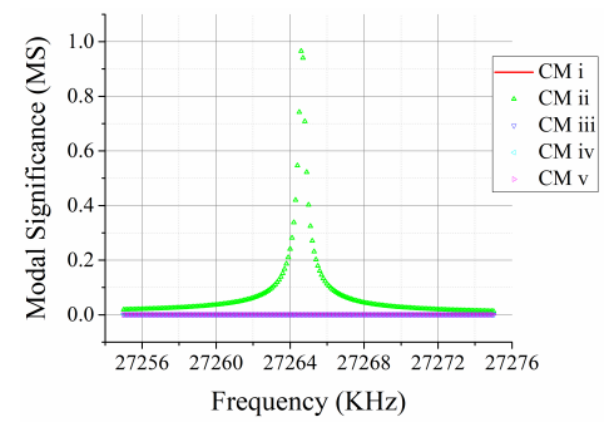

Fig. 10. MS curves of the first $5 \mathrm{CMs}$ of the one-coil scattering system.

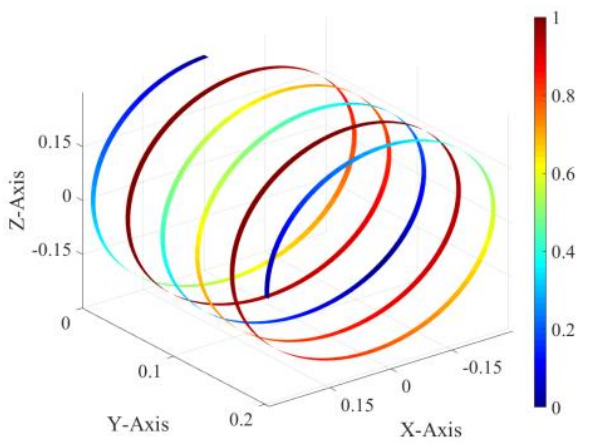

Fig. 11. Modal current magnitude distribution of the two-order externally resonant CM ii working at $27.2646 \mathrm{MHz}$.

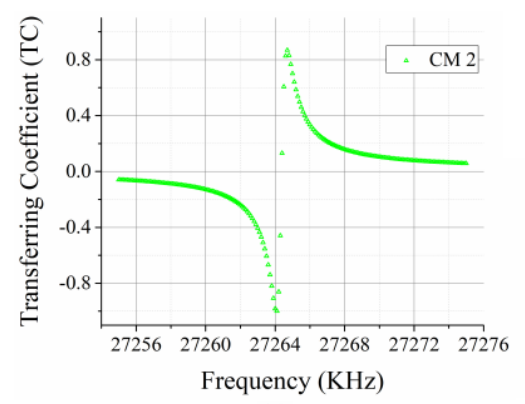

(a)

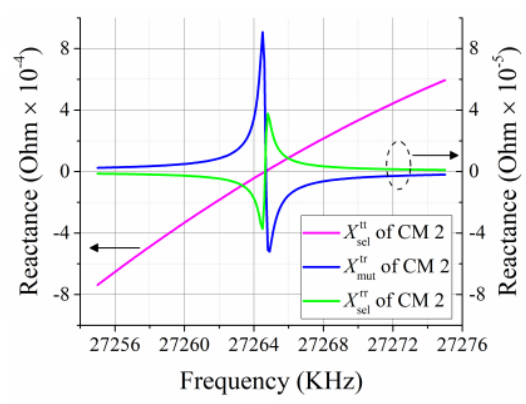

(b)

Fig. 12. (a) TC curve and (b) reactance curves associated with the CM 2 of the two-coil transferring system working in the frequency band used in Fig. 10. 


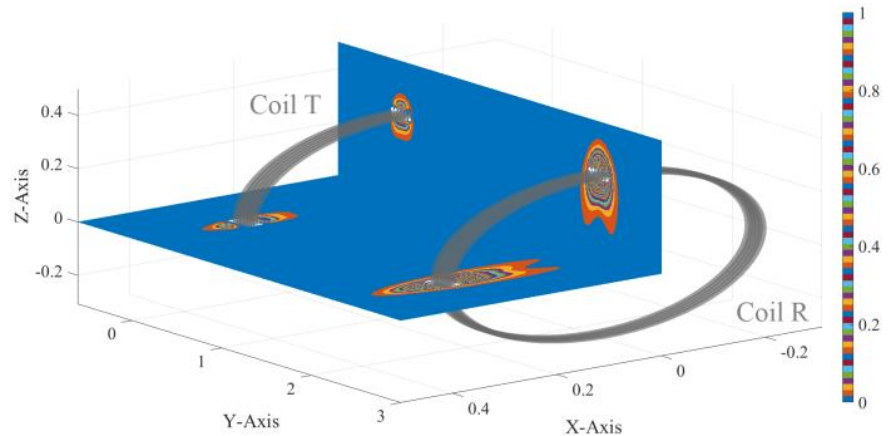

Fig. 13. For the co-resonant CM 2 working at $27.2646 \mathrm{MHz}$, its time-average magnetic energy density distributing on $\mathrm{xOy}$ and $\mathrm{yOz}$ planes.

The Fig. 12(b) implies that the CM 2 is co-resonant at 27.2646 MHz. The time-average magnetic energy density distribution of the co-resonant CM 2 working at co-resonance frequency $27.2646 \mathrm{MHz}$ is shown in Fig. 13.

By comparing the Figs. 12(a)\&13 with the previous Figs. $4 \& 6$, it is not difficult to find out that:

C6 the one-order co-resonant mode has a higher wireless power transferring efficiency than the two-order co-resonant mode.

In fact, this conclusion can be generalized to the comparison between any lower-order and higher-order co-resonant modes.

\section{On Transferring Frequency - Part III}

The above-mentioned two-coil transferring system is constituted by two completely same coils, which have the same geometrical size and external-resonance frequencies 10.8988 $\mathrm{MHz}$ (one order) and $27.2646 \mathrm{MHz}$ (two order) etc. Now, we replace the coil $\mathrm{R}$ with another different coil, whose geometrical size is "radius $30 \mathrm{~cm}$, height $8.15 \mathrm{~cm}$, and turns 2.138 " and one-order external-resonance frequency is $27.2646 \mathrm{MHz}$ and higher-order external resonance frequencies are larger than 27.2646 MHz. We calculate the CMs of the new two-coil transferring system working around frequencies $10.8988 \mathrm{MHz}$ and $27.2646 \mathrm{MHz}$, and we show the corresponding TC and reactance curves in Fig. 14 and Fig. 15 respectively.

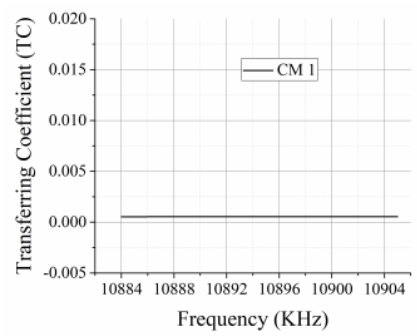

(a)

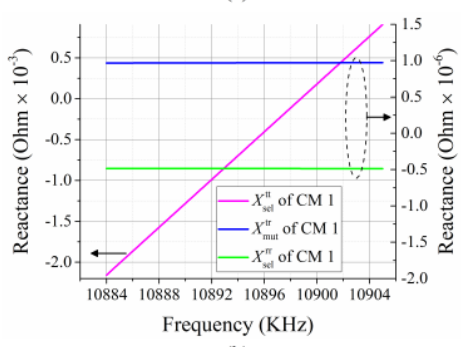

(b)

Fig. 14. (a) TC curve and (b) reactance curves associated with the CM 1 of the new two-coil transferring system working around $10.8988 \mathrm{MHz}$.

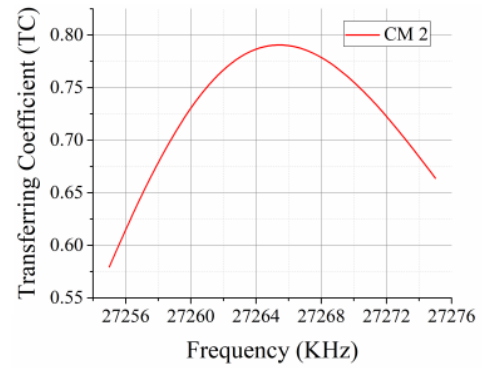

(a)

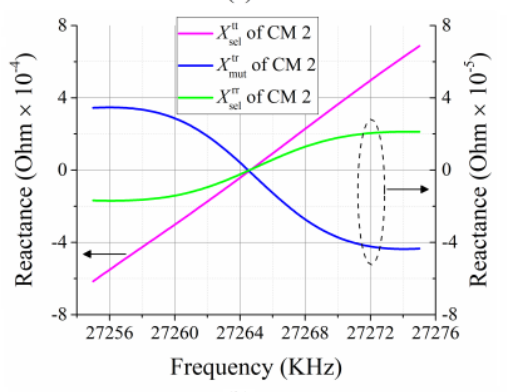

(b)

Fig. 15. (a) TC curve and (b) reactance curves associated with the CM 2 of the new two-coil transferring system working around 27.2646 MHz.

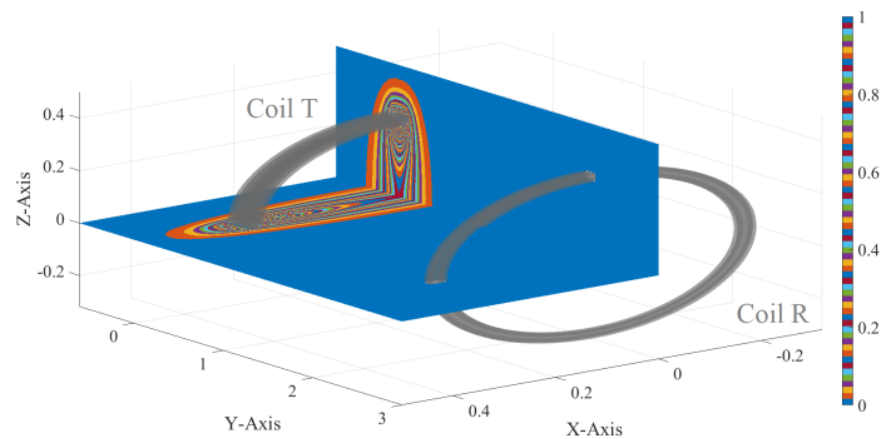

(a)

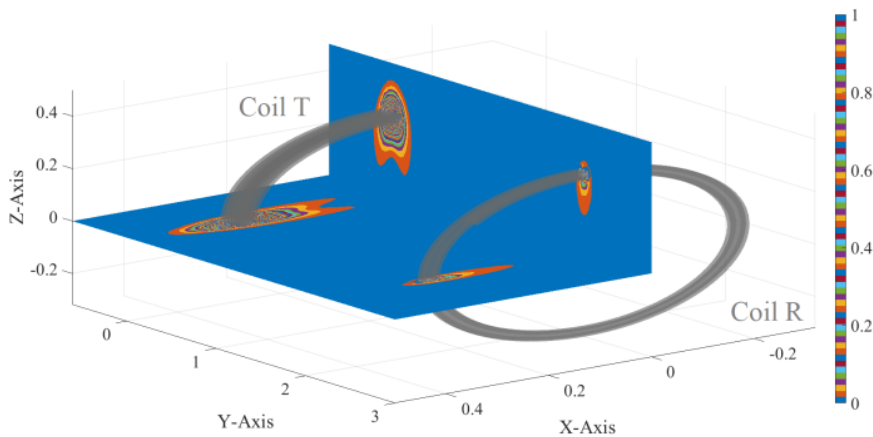

(b)

Fig. 16. Time-average magnetic energy density distributions of (a) the CM 1 working at $10.8988 \mathrm{MHz}$ and (b) the CM 2 working at $27.2646 \mathrm{MHz}$.

From Figs. 14 and 15, it is easy to find out that: the coil T working at CM 1 is self-resonant at $10.8988 \mathrm{MHz}\left(X_{\text {sel }}^{\mathrm{tt}}=0\right)$, but $10.8988 \mathrm{MHz}$ is not the co-resonance frequency of the new two-coil transferring system $\left(X_{\text {mut }}^{\mathrm{tr}}, X_{\text {sel }}^{\mathrm{rr}} \neq 0\right)$, and the TC is very small at $10.8988 \mathrm{MHz} ; 27.2646 \mathrm{MHz}$ is the co-resonance frequency of the new two-coil transferring system, and the TC curve achieves its local maximum at 27.2646 MHz.

Based on the above observations and the comparisons among Figs. 4, 12(a), and 15(a), we can conclude that:

C7 When only one coil is self-resonant, the whole system cannot efficiently transfer power wirelessly. 
C8 When two coils have the same external-resonance frequency, and the whole two-coil transferring system works at the frequency, the system can transfer power wirelessly even if the orders of the frequencies are different.

C9 The same-order co-resonance is more desired than the different-order co-resonance in WPT application.

To visualize the above conclusions, we show the corresponding time-average magnetic energy density distributions in Fig. 16.

\section{On Transferring Distance}

In the above all discussions, the distance between coil $\mathrm{T}$ and coil $\mathrm{R}$ is $2 \mathrm{~m}$. In this sub-section, we study how the distance influences transferring efficiency.

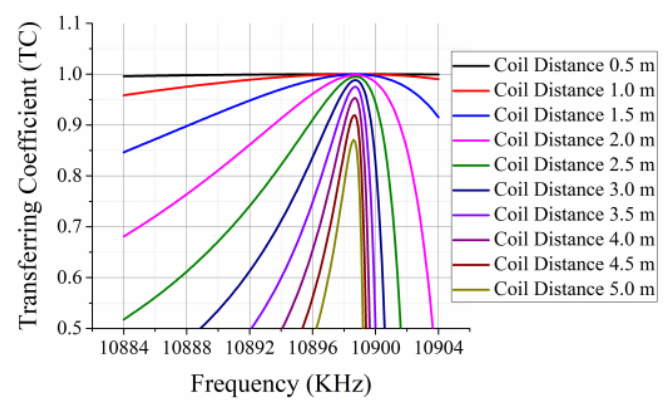

Fig. 17. TC curves of the one-order CMs corresponding to the different coil-separation distances.

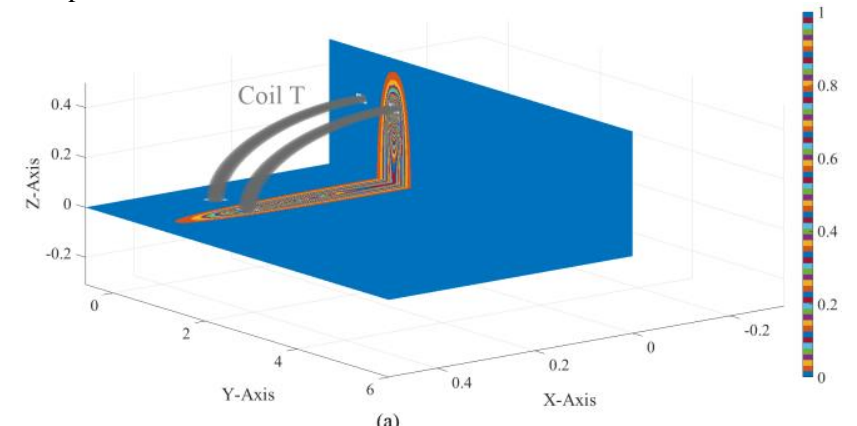

(a)

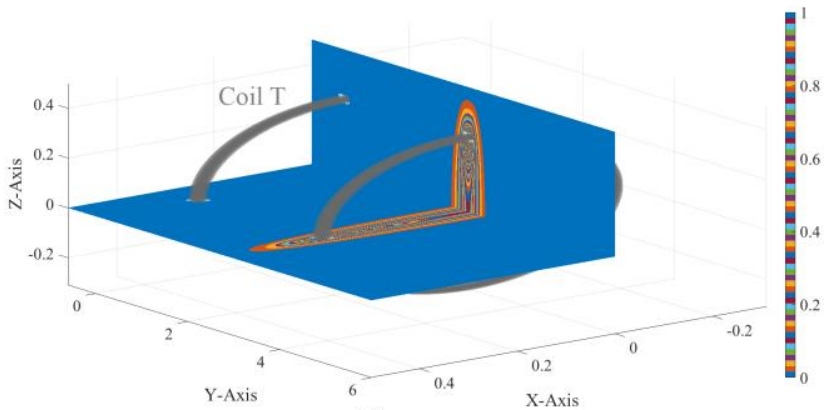

(b)

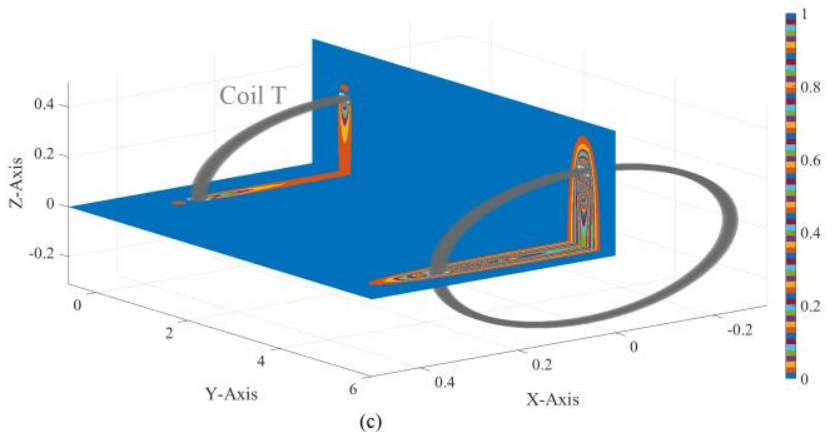

Fig. 18. Time-average magnetic energy density distributions corresponding to coil-separation distances (a) $0.5 \mathrm{~m}$, (b) $2.5 \mathrm{~m}$, and (c) $5.0 \mathrm{~m}$.
We use the WEP-CMT to calculate the two-coil transferring systems with a series of different coil-separation distances (where the coil $\mathrm{T}$ and coil $\mathrm{R}$ are the same as the ones considered in Sec. IV), and show the TC curves of the one-order CMs corresponding to the different coil-separation distances in Fig. 17.

The Fig. 17 clearly implies the following expected conclusion.

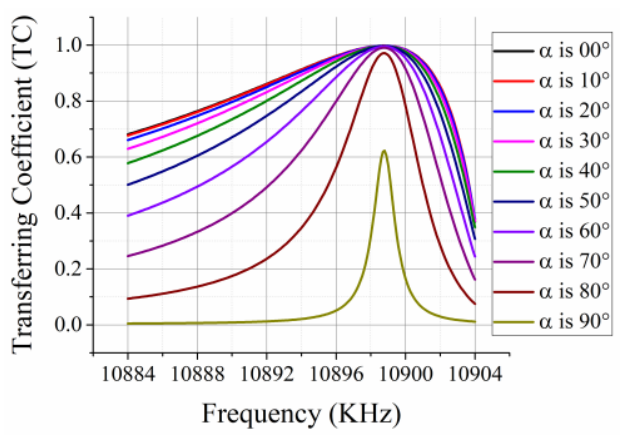

Fig. 19. TC curves of the one-order CMs corresponding to different coil-coil angle $\alpha$.

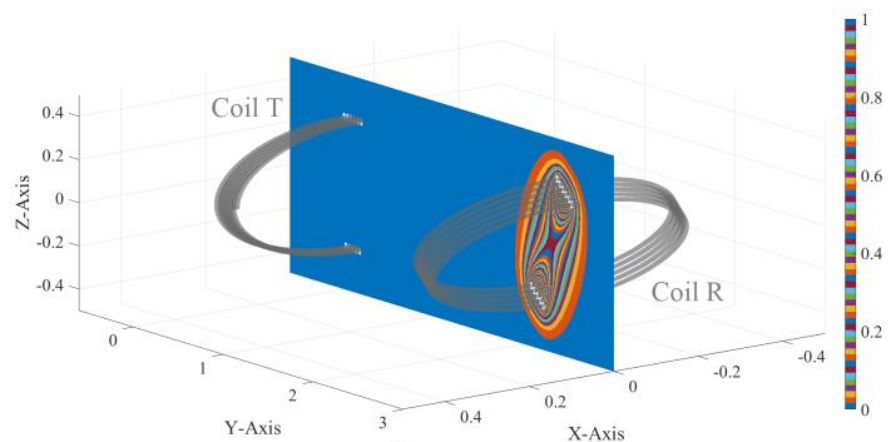

(a)

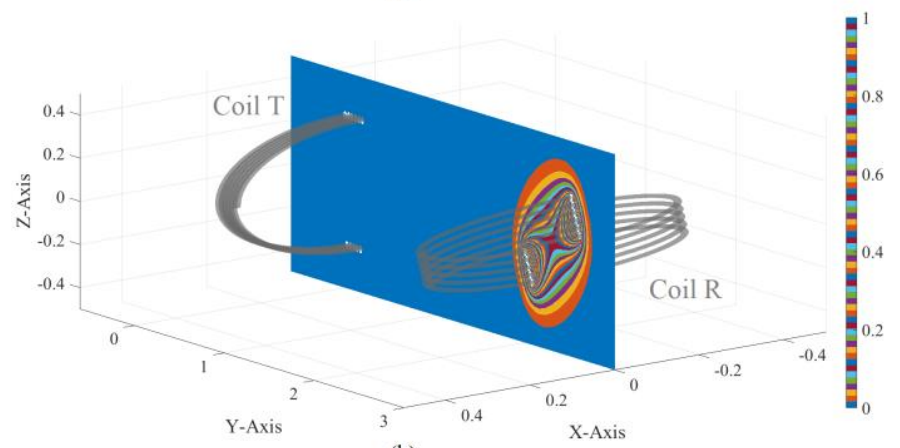

(b)

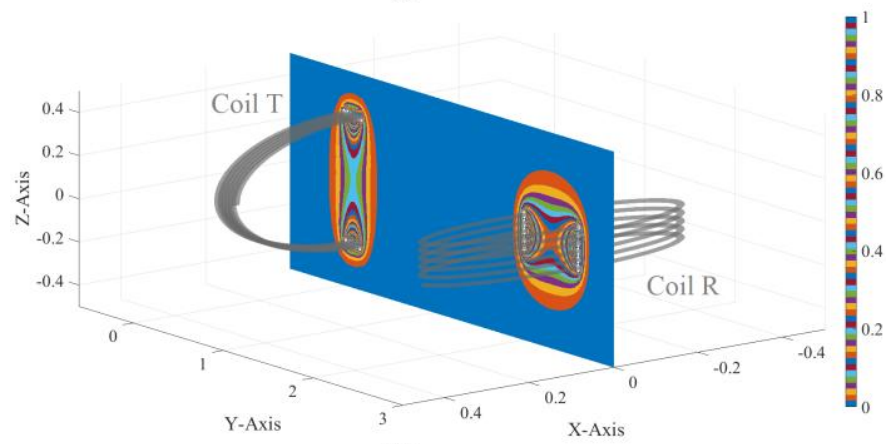

(c)

Fig. 20. Time-average magnetic energy density distributions corresponding to (a) $\alpha=30^{\circ}$, (b) $\alpha=60^{\circ}$, and (c) $\alpha=90^{\circ}$. 
C10 When two same coils are placed axially, the co-resonance frequency of the two-coil transferring system is almost independent of the distance between the coils, and the TC of the system is a monotonically decreasing function about the distance.

To visually exhibit the above conclusion, we show the time-average magnetic energy density distributions corresponding to distances $0.5 \mathrm{~m}, 2.5 \mathrm{~m}$, and $5.0 \mathrm{~m}$ in Fig. 18 .

\section{E. On Transferring Direction}

In this sub-section, we employ the WEP-CMT to study how the relative direction between coil $\mathrm{T}$ and coil $\mathrm{R}$ influences the transferring efficiency. The angle between the axis of the two coils is denoted as $\alpha$ (in degree). We calculate the CMs of the transferring systems with $\alpha=0^{\circ}, 10^{\circ}, 20^{\circ}, \ldots, 90^{\circ}$, and show the corresponding TC curves in Fig. 19.

Evidently, Fig. 19 clearly implies the following expected conclusion

C11 When two same coils are placed with an angle $\alpha$, the co-resonance frequency of the two-coil transferring system is almost independent of $\alpha$, and the TC of the system is a monotonically decreasing function about $\alpha$.

To visually exhibit the above conclusion, we show the time-average magnetic energy density distributions corresponding to $\alpha=30^{\circ}, 60^{\circ}$, and $90^{\circ}$ in Fig. 20.

\section{F. On Multi-coil Transferring System}

In the above discussions, only some typical two-coil transferring systems are analyzed. In this sub-section, we further apply the WEP-CMT to some typical multi-coil transferring systems.

Firstly, we consider the three-coil transferring system whose three coils have the same geometrical size and are coaxially placed with mutual distance $2 \mathrm{~m}$. We use WEP-CMT to calculate the CMs of the transferring system, and show the TC curve corresponding to CM 1 in Fig. 21. For the CM 1 working at the optimally transferring frequency 10.8872, its time-average magnetic energy density distribution is shown in Fig. 22.

Secondly, we consider the three-coil transferring system whose three coils have the same geometrical size but are misaligned. We use WEP-CMT to calculate the CMs of the transferring system, and show the TC curve corresponding to CM 1 in Fig. 23. For the CM 1 working at the optimally transferring frequency 10.8724 , its time-average magnetic energy density distribution is shown in Fig. 24.

By comparing the Figs. $21 \& 23$ with the previous Figs. $4 \& 17 \& 19$, it is not difficult to find out that:

C12 The optimally transferring frequency of three-coil transferring system is usually different from the optimally transferring frequency of classical two-coil transferring system.

The reason leading to this conclusion is that the external-resonance frequency of scattering system $\mathrm{R}$ (discussed previously) is different from the external-resonance frequency of R1-R2 scattering system (discussed here). In fact, the conclusion is also applicable to the multi-coil transferring systems constituted by $N(\geq 3)$ coils.

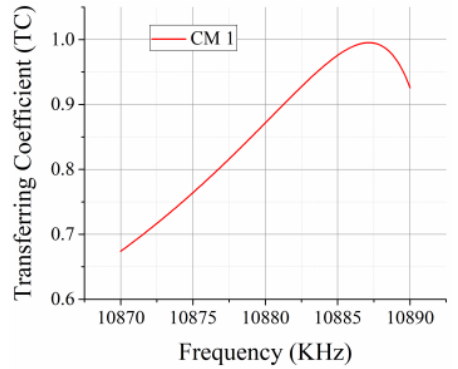

Fig. 21. TC curve associated with the CM 1 of the transferring system constituted by three coaxially placed coils.

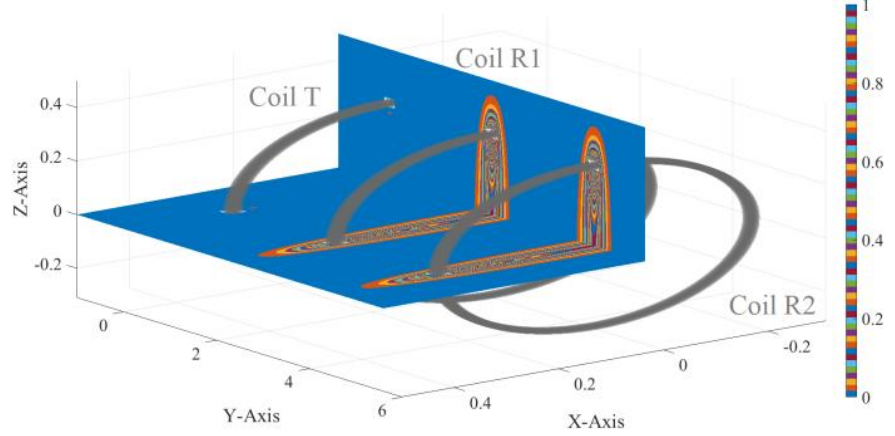

Fig. 22. For the CM 1 working at $10.8872 \mathrm{MHz}$ (shown in Fig. 21), its time-average magnetic energy density distributing on $\mathrm{xOy}$ and $\mathrm{yOz}$ planes.

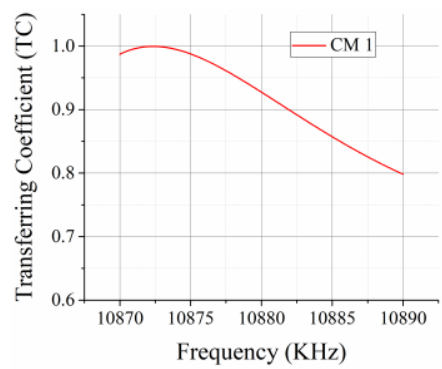

Fig. 23. TC curve associated with the CM 1 of the transferring system constituted by three misaligned coils.

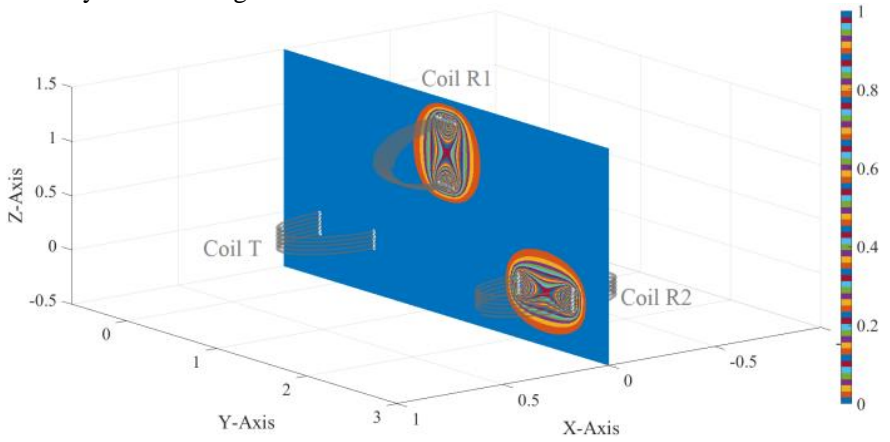

Fig. 24. For the CM 1 working at $10.8724 \mathrm{MHz}$ (shown in Fig. 23), its time-average magnetic energy density distributing on $\mathrm{xOy}$ and yOz planes.

\section{G. On Surrounding Environment}

In this sub-section, we use WEP-CMT to simply study how surrounding environment influences the WPT process.

We integrate a square thin metallic plane into the two-coil transferring system considered in Sec. IV. We use WEP-CMT to calculate the CMs of the transferring systems with three different planes having side-lengths $0.6 \mathrm{~m}, 1.2 \mathrm{~m}$, and $2.4 \mathrm{~m}$, and show the TC curves corresponding to the one-order CMs in Fig. 25. We illustrate the time-average magnetic energy density distributions of the optimally transferring modes shown in Fig. 25 as Fig. 26. 


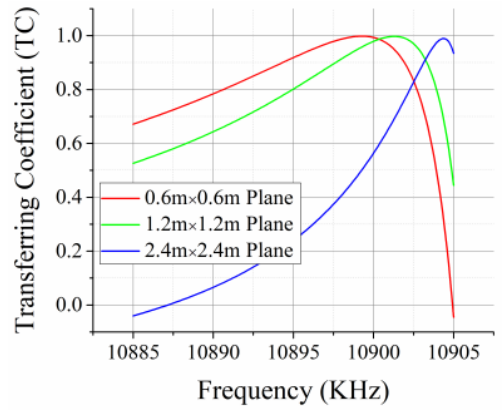

Fig. 25. TC curves of the one-order CMs corresponding to different plane sizes.

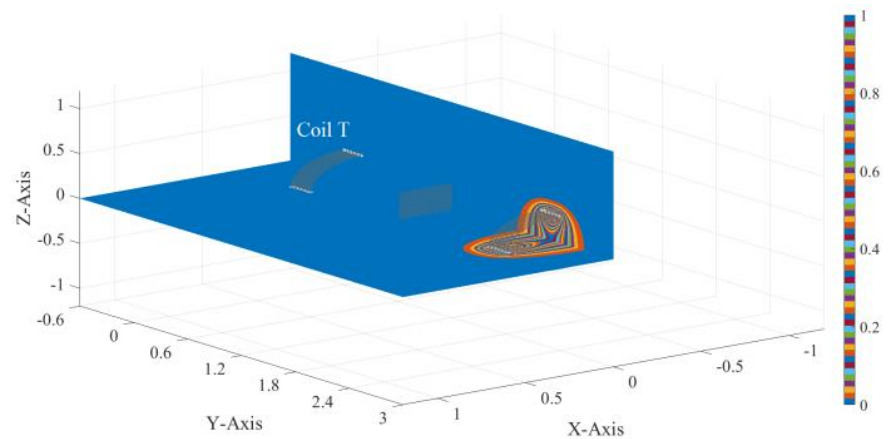

(a)

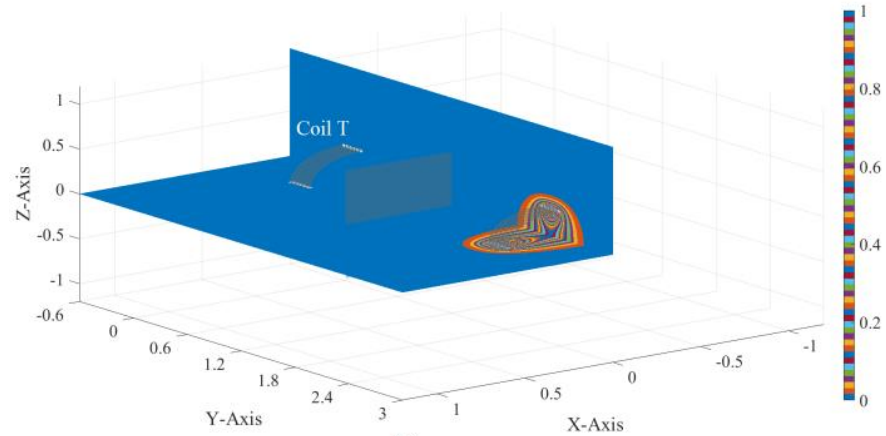

(b)

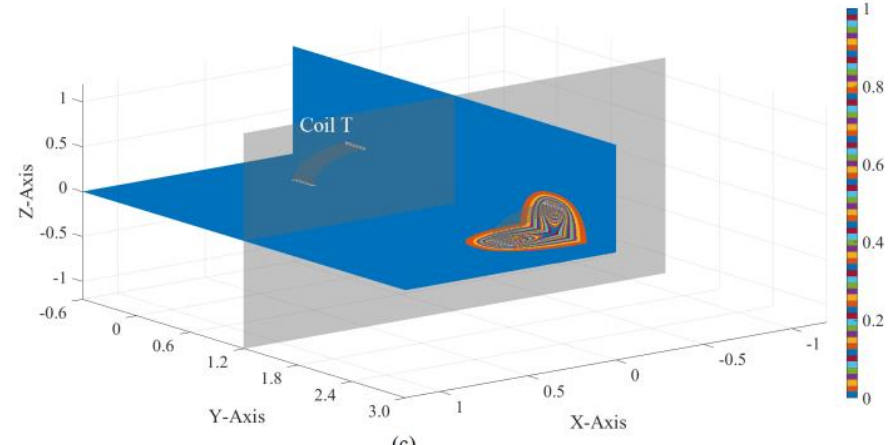

(c)

Fig. 26. Time-average magnetic energy density distributions of the optimally transferring modes with (a) $0.6 \mathrm{~m} \times 0.6 \mathrm{~m}$ plane, (b) $1.2 \mathrm{~m} \times 1.2 \mathrm{~m}$ plane, and (c) $2.4 \mathrm{~m} \times 2.4 \mathrm{~m}$ plane.

From the above Figs. 25 and 26, it is not difficult to find out the following conclusion.

C13 For the above-considered metallic planes placed between the coils, they almost don't hinder the power wirelessly transferred from coil $\mathrm{T}$ to coil $\mathrm{R}$, but they indeed influence the optimally transferring frequency, and the altered optimally transferring frequency depends on the geometrical size of the plane.
The reason leading to the conclusion is that the external-resonance frequency of scattering system $\mathrm{R}$ is different from the external-resonance frequency of R-Plane scattering system. In fact, the above conclusion can also be generalized to the other kinds of surrounding environments.

\section{CONCLUSIONS}

Focusing on the classical two-coil transferring system, which is constituted by a transmitting coil $\mathrm{T}$ (driven by locally impressed driver) and a receiving coil $\mathrm{R}$ (loaded by perfectly matched load), the WEP governing the WPT process of the transferring system is derived from Maxwell's vector field theory, and doesn't employ any scalar circuit model. The WEP gives the WPT process a very clear physical picture: during the WPT process, the driving power from driver to coil $\mathrm{T}$ acts as the source to sustain a steady WPT, and the driving power is finally transformed into two parts - a part is dissipated by coil $\mathrm{T}$ and the other part is wirelessly transferred from coil $\mathrm{T}$ to coil $\mathrm{R}$. Based on the WEP, a novel concept of TC, which is equal to the ratio of time-average transferred power to time-average driving power, is introduced to quantitatively describe the transferring efficiency of the transferring system. From a relatively mathematical viewpoint, the design process for transferring system is to search for the physically realizable objective modes which can maximize TC, and the objective modes are called optimally transferring modes.

The WEP clearly reveals the fact that: the working mechanisms of scattering systems and transferring systems are different from each other. The fact clearly exposes the problem that: the conventional CMT for scattering systems cannot be directly applied to transferring systems. To resolve this problem, this paper, under WEP framework, generalizes the conventional scattering CMT to a novel transferring CMT. The WEP-based transferring CMT (transferring WEP-CMT) can be employed to search for the optimally transferring modes. Specifically, the transferring WEP-CMT constructs a set of energy-decoupled CMs by orthogonalizing DPO, and the optimally transferring modes can be found in the obtained $\mathrm{CM}$ set.

The validity of the transferring WEP-CMT is verified by applying it to the classical two-coil transferring system. After the verification, the WEP-CMT is further applied to the numerical experiments for some typical variants of the classical two-coil transferring system, and then some valuable conclusions are obtained as summarized below.

C1 The larger TC is, the more efficiently "driver drives coil T" and "power is transferred from coil T to coil R" and "coil $\mathrm{R}$ converts the transferred power into its self-power".

$\mathrm{C} 2$ When TC is maximized, coils $\mathrm{T}$ and $\mathrm{R}$ are not only self-resonant but also co-resonant. The co-resonant mode works at the optimally transferring state.

C3 Co-resonance is usually called magnetic resonance. The so-called magnetic resonance is a special case of classical electric-magnetic resonance.

C4 Ci-resonance is different from co-resonance. The ci-resonant mode cannot guarantee an efficient WPT from coil $\mathrm{T}$ to coil $\mathrm{R}$. 
C5 When coils $\mathrm{T}$ and $\mathrm{R}$ are the same, the co-resonance frequency of two-coil transferring system equals to the $e x$ ternal-resonance frequency of one-coil scattering system.

C6 When coils $\mathrm{T}$ and $\mathrm{R}$ are the same, the lower-order co-resonant mode has a larger TC than the higher-order co-resonant mode.

C7 When coils $\mathrm{T}$ and $\mathrm{R}$ are different, and only one coil is resonant, the system usually cannot efficiently transfer power from coil $\mathrm{T}$ to coil $\mathrm{R}$.

C8 When different coil $\mathrm{T}$ and coil $\mathrm{R}$ work at a same resonance frequency, the system can transfer power wirelessly even if the resonance orders of the coils are different.

C9 For a two-coil transferring system, the same-order co-resonance is usually more desired than the different-order co-resonance in WPT application.

C10 The co-resonance frequency of two same and aligned coils is almost independent of coil distance, and TC is a monotonically decreasing function about the distance.

C11 The co-resonance frequency of two same and misaligned coils is almost independent of the relative angle, and TC is a monotonically decreasing function about the angle.

C12 The optimally transferring frequency of $N$-coil $(N \geq 3)$ system depends on the external-resonance frequency of the scattering system constituted by $N-1$ receiving coils.

C13 The optimally transferring frequency of the system in environment depends on the external-resonance frequency of the Environment-Receiving-coil scattering system.

In fact, the above conclusions also effectively establish some connections "between wireless power transferring problem and conventional scattering problem", "between co-resonance and conventional external-resonance", and "between so-called magnetic resonance and classical electric-magnetic resonance".

\section{APPENDICES}

In this section, some detailed derivations and formulations related to this paper are provided.

A. Rigorous Derivation for Work-Energy Principle (1) and More Detailed Decomposition for Driving Power

Based on the discussions in Sec. II, the tangential components of electric fields $\mathcal{E}_{\text {driv }}+\mathcal{E}_{\mathrm{t}}+\mathcal{E}_{\mathrm{r}}$ and $\mathcal{E}_{\mathrm{t}}+\mathcal{E}_{\mathrm{r}}$ are zero on $S_{\mathrm{t}}$ and $S_{\mathrm{r}}$ respectively, so

$$
\begin{aligned}
& \overbrace{\left\langle\boldsymbol{J}_{\mathrm{t}}, \boldsymbol{\mathcal { E }}_{\text {driv }}\right\rangle_{S_{\mathrm{t}}}}^{\mathcal{P}_{\text {div }}}=\left\langle\boldsymbol{J}_{\mathrm{t}},-\mathcal{E}_{\mathrm{t}}-\mathcal{E}_{\mathrm{r}}\right\rangle_{S_{\mathrm{t}}}+\overbrace{\left\langle\boldsymbol{J}_{\mathrm{r}},-\mathcal{E}_{\mathrm{t}}-\mathcal{E}_{\mathrm{r}}\right\rangle_{S_{\mathrm{r}}}}^{0}
\end{aligned}
$$

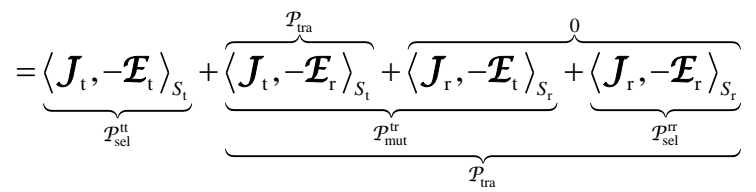

The physical meanings of $\mathcal{P}_{\text {sel }}^{\text {tt }}$ and $\mathcal{P}_{\text {tra }}$ had been carefully discussed in Sec. II. The $\mathcal{P}_{\text {mut }}^{\text {tr }}$ and $\mathcal{P}_{\text {sel }}^{\text {rr }}$ can be similarly discussed, and they are respectively called mutual-power between coil $\mathrm{T}$ and coil $\mathrm{R}$ and self-power of coil $\mathrm{R}$.
B. Deriving Transformation (6) from Electric Field Integral Equation (5)

Expanding the currents in (5) in terms of some proper basis functions and testing (5) with Galerkin's method, (5) is discretized as follows:

$$
\mathrm{Z}_{\mathrm{rt}} \cdot \mathrm{J}_{\mathrm{t}}+\mathrm{Z}_{\mathrm{rr}} \cdot \mathrm{J}_{\mathrm{r}}=0
$$

The elements of $Z_{\mathrm{rt}}$ are $\left.\left[Z_{\mathrm{rt}}\right]_{\xi \zeta}=<\boldsymbol{b}_{\mathrm{r} ; \xi},-j \omega \mu_{0} \mathcal{L}_{0}\left(\boldsymbol{b}_{\mathrm{t}, \zeta}\right)\right\rangle_{\mathrm{S}_{\mathrm{r}}}$, and the elements of $Z_{\mathrm{rr}}$ are $\left.\left[Z_{\mathrm{rr}}\right]_{\xi \zeta}=<\boldsymbol{b}_{\mathrm{r}, \xi},-j \omega \mu_{0} \mathcal{L}_{0}\left(\boldsymbol{b}_{\mathrm{r}, \zeta}\right)\right\rangle_{S_{\mathrm{r}}}$. By solving (12), the transformation $\mathrm{J}_{\mathrm{r}}=\mathrm{T} \cdot \mathrm{J}_{\mathrm{t}}$ given in (6) is immediately obtained, where $T=-Z_{\mathrm{rr}}^{-1} \cdot Z_{\mathrm{rt}}$.

C. Deriving Matrix Quadratic Form (7) from Driving Power Operator (4)

By expanding the currents in (4), the (4) is discretized as follows:

$$
P_{\text {driv }}=\left[\begin{array}{l}
\mathrm{J}_{\mathrm{t}} \\
\mathrm{J}_{\mathrm{r}}
\end{array}\right]^{\dagger} \cdot\left[\begin{array}{ll}
\mathrm{P}_{\mathrm{tt}} & \mathrm{P}_{\mathrm{tr}} \\
\mathrm{O}_{\mathrm{rt}} & \mathrm{O}_{\mathrm{rr}}
\end{array}\right] \cdot\left[\begin{array}{l}
\mathrm{J}_{\mathrm{t}} \\
\mathrm{J}_{\mathrm{r}}
\end{array}\right]
$$

where the sub-matrices $\mathrm{O}_{\mathrm{rt}}$ and $\mathrm{O}_{\mathrm{rr}}$ are zero matrices with proper row number and column number, and the elements of sub-matrix $\mathrm{P}_{\mathrm{tt}}$ are $\left.(1 / 2)<\boldsymbol{b}_{\mathrm{t} ; \xi}, j \omega \mu_{0} \mathcal{L}_{0}\left(\boldsymbol{b}_{\mathrm{t} ; 5}\right)\right\rangle_{s_{\mathrm{t}}}$, and the elements of sub-matrix $\mathrm{P}_{\mathrm{tr}}$ are $(1 / 2)<\boldsymbol{b}_{\mathrm{t} ; \xi}, j \omega \mu_{0} \mathcal{L}_{0}\left(\boldsymbol{b}_{\mathrm{r} ; \zeta}\right)>_{S_{\mathrm{t}}}$.

Substituting transformation (6) into (13), matrix quadratic form (7) is immediately obtained, in which

$$
P_{\text {driv }}=\left[\begin{array}{c}
I_{t} \\
T
\end{array}\right]^{\dagger} \cdot\left[\begin{array}{ll}
P_{t t} & P_{t r} \\
O_{r t} & O_{r r}
\end{array}\right] \cdot\left[\begin{array}{c}
I_{t} \\
T
\end{array}\right]
$$

where $I_{t}$ is the unit matrix with the same dimension as the row number of $\mathrm{J}_{\mathrm{t}}$.

In addition, the matrix $P_{\text {tra }}^{+}$used in (9) is as that $\mathrm{P}_{\text {tra }}^{+}=\left(\mathrm{P}_{\text {tra }}+\mathrm{P}_{\text {tra }}^{\dagger}\right) / 2$, in which

$$
P_{\text {tra }}=\left[\begin{array}{c}
I_{t} \\
T
\end{array}\right]^{\dagger} \cdot\left[\begin{array}{ll}
O_{t t} & P_{t r} \\
O_{r t} & O_{\text {rr }}
\end{array}\right] \cdot\left[\begin{array}{c}
I_{t} \\
T
\end{array}\right]
$$

where sub-matrices $P_{\mathrm{tr}}, \mathrm{O}_{\mathrm{rt}}$, and $\mathrm{O}_{\mathrm{rr}}$ are the same as the ones used in (13) and (14), and submatrix $\mathrm{O}_{\mathrm{tt}}$ is a zero matrix with proper row number and column number, and the derivation for (15) is similar to the derivation for (14).

\section{Resistance and Reactance}

For the convenience of Sec. IV and using the convention of [50], we define resistances $R_{\mathrm{driv}}, R_{\mathrm{sel}}^{\mathrm{tt}}, R_{\mathrm{mut}}^{\mathrm{tr}}, R_{\mathrm{sel}}^{\mathrm{rr}}$, and $R_{\mathrm{tra}}$ as the powers $\operatorname{Re}\left\{P_{\text {driv }}\right\}, \operatorname{Re}\left\{P_{\text {sel }}^{\mathrm{tr}}\right\}, \operatorname{Re}\left\{P_{\text {mut }}^{\mathrm{tr}}\right\}, \operatorname{Re}\left\{P_{\text {sel }}^{\mathrm{rr}}\right\}$, and $\operatorname{Re}\left\{P_{\text {tra }}\right\}$ divided by $\left.(1 / 2)<\boldsymbol{J}_{\mathrm{t}}, \boldsymbol{J}_{\mathrm{t}}\right\rangle_{S_{\mathrm{t}}}$, and we define reactances $X_{\text {driv }}, X_{\text {sel }}^{\mathrm{tt}}, X_{\text {mut }}^{\mathrm{tr}}, X_{\text {sel }}^{\mathrm{rr}}$, and $X_{\text {tra }}$ as the powers $\operatorname{Im}\left\{P_{\text {driv }}\right\}, \operatorname{Im}\left\{P_{\text {sel }}^{\mathrm{tc}}\right\}, \operatorname{Im}\left\{P_{\text {mut }}^{\mathrm{tr}}\right\}, \operatorname{Im}\left\{P_{\text {sel }}^{\mathrm{rr}}\right\}$, and $\operatorname{Im}\left\{P_{\text {tra }}\right\}$ divided by $\left.(1 / 2)<\boldsymbol{J}_{\mathrm{t}}, \boldsymbol{J}_{\mathrm{t}}\right\rangle_{S_{\mathrm{t}}}$. 


\section{REFERENCES}

[1] M. Hutin and M. Leblanc, "Transformer system for electric railways," U.S. patent 527857, Oct. 23, 1894.

[2] N. Tesla, "High frequency oscillators for electro-therapeutic and other purposes (classic paper)," Proc. IEEE, vol. 87, no. 7, Jul. 1999.

[3] N. Tesla, The Transmission of Electric Energy Without Wires (The Thirteenth Anniversary Number of the Electrical World and Engineer)., McGraw-Hill, Mar. 1904.

[4] N. Tesla, "Apparatus for transmitting electrical energy," U.S. Patent 1119732, Dec. 1, 1914.

[5] W. C. Brown, "The history of wireless power transmission," Solar Energy, vol. 56, no. 1, pp. 3-21, 1996.

[6] J. Garnica, R. A. Chinga, and J. Lin, "Wireless power transmission: From far field to near field," Proc. IEEE, vol. 101, no. 6, pp. 1321-1331, Jun. 2013

[7] W. C. Brown, "The history of power transmission by radio waves," IEEE Trans. Microw. Theory Tech., vol. MTT-32, no. 9, pp. 1230-1242, Sep. 1984.

[8] N. Shinohara, Wireless Power Transfer via Radiowaves. London: ISTE Ltd, 2014.

[9] Q. Zhang, W. Fang, Q. Liu, J. Wu, P. Xia, and L. Yang, "Distributed laser charging: A wireless power transfer approach," IEEE Internet Thing J., vol. 5, no. 5, pp. 3853-3864, Oct. 2018.

[10] K. Jin and W. Zhou, "Wireless laser power transmission: A review of recent progress," IEEE Trans. Power Electron., vol. 34, no. 4, pp. 3842-3859, Apr. 2019.

[11] B. Lenaerts and R. Puers, Omnidirectional Inductive Powering for Biomedical Implants. Berlin: Springer, 2009.

[12] G. Zulauf and J. M. Rivas-Davila, "Single-tune air-core coils for high-frequency inductive wireless power transfer," IEEE Trans. Power Electron., vol. 35, no. 3, pp. 2917-2932, Mar. 2020.

[13] A. Koran and K. Badran, "Adaptive frequency control of a sensorless-receiver inductive wireless power transfer system based on mixed-compensation topology," IEEE Trans. Power Electron., vol. 36, no. 1, pp. 978-990, Jan. 2021.

[14] M. Tamura, Y. Naka, K. Murai, and T. Nakata, "Design of a capacitive wireless power transfer system for operation in fresh water," IEEE Trans. Microw. Theory Tech., vol. 66, no. 12, pp. 5873-5884, Dec. 2018.

[15] S. Sinha, A. Kumar, B. Regensburger, and K. K. Afridi, "A new design approach to mitigating the effect of parasitics in capacitive wireless power transfer systems for electric vehicle charging," IEEE Trans. Transp. Electrific., vol. 5, no. 4, pp. 1040-1059, Dec. 2019.

[16] M. Tudela-Pi, L. Becerra-Fajardo, A. García-Moreno, J. Minguillon, and A. Ivorra, "Power transfer by volume conduction: In vitro validated analytical models predict DC powers above $1 \mathrm{~mW}$ in injectable implants," IEEE Access, vol. 8, pp. 37808-37820, 2020.

[17] R. Sedehi, D. Budgett, J. C. Jiang, Z. Y. Xia, X. Dai, A. P. Hu, and D. McCormick, "A wireless power method for deeply implanted biomedical devices via capacitively coupled conductive power transfer," IEEE Trans. Power Electron., vol. 36, no. 2, pp. 1870-1882, Feb. 2021.

[18] A. Kurs, A. Karalis, R. Moffatt, J. D. Joannopoulos, P. Fisher, and M. Soljačić, "Wireless power transfer via strongly coupled magnetic resonances," Science, vol. 317, no. 5834, pp. 83-86, Jul. 2007.

[19] A. Karalis, J. D. Joannopoulos, and M. Soljačić, "Efficient wireless non-radiative midrange energy transfer," Ann. Phys., vol. 323, pp. 34-48, Apr. 2007.

[20] S. Y. R. Hui, W. X. Zhong, and C. K. Lee, "A critical review of recent progress in mid-range wireless power transfer," IEEE Trans. Power Electron., vol. 29, no. 9, pp. 4500-4511, Sep. 2014.

[21] S. Y. R. Hui, "Magnetic resonance for wireless power transfer [A look back]," IEEE Power Electron. Mag., vol. 3, no. 1, pp. 14-31, Mar. 2016.

[22] J. I. Agbinya, Wireless Power Transfer, 2nd. Denmark: River Publishers, 2016.

[23] W. X. Zhong, D. H. Xu, and S. Y. R. Hui, Wireless Power Transfer: Between Distance and Efficiency. Singapore: Springer, 2020.

[24] On line: https://en.wikipedia.org/wiki/Tesla_coil.

[25] S. Y. R. Hui, "Planar wireless charging technology for portable electronic products and Qi," Proc. IEEE, vol. 101, no. 6, pp. 1290-1301, Jun. 2013.

[26] C. T. Rim and C. Mi, Wireless Power Transfer for Electric Vehicles and Mobile Devices. Hoboken: John Wiley \& Sons, 2017.

[27] A. Trivino-Cabrera, J. M. González-González, and J. A. Aguado, Wireless Power Transfer for Electric Vehicles: Foundations and Design Approach. Switzerland: Springer, 2020.
[28] T. J. Sun, X. Xie, and Z. H. Wang, Wireless Power Transfer for Medical Microsystems. New York: Springer, 2013.

[29] G. Yilmaz and C. Dehollain, Wireless Power Transfer and Data Communication for Neural Implants - Case Study: Epilepsy Monitoring. Switzerland: Springer, 2017.

[30] K. Türe, C. Dehollain, and F. Maloberti, Wireless Power Transfer and Data Communication for Intracranial Neural Recording Applications. Switzerland: Springer, 2020.

[31] W. Niu, W. Gu, and J. Chu, "Analysis and experimental results of frequency splitting of underwater wireless power transfer," J. Eng., vol. 7, pp. 385-390, Jul. 2017.

[32] R. Hasaba, K. Okamoto, S. Kawata, K. Eguchi, and Y. Koyanagi, "Magnetic resonance wireless power transfer over $10 \mathrm{~m}$ with multiple coils immersed in seawater," IEEE Trans. Microw. Theory Tech., vol. 67, no. 11, pp. 4505-4513, Jul. 2019.

[33] L. Wang, X. B. Li, S. Raju, and C. P. Yue, "Simultaneous magnetic resonance wireless power and high-speed data transfer system with cascaded equalizer for variable channel compensation," IEEE Trans. Power Electron., vol. 34, no. 12, pp. 11594-11604, Dec. 2019.

[34] P. F. Wu, F. Xiao, H. P. Huang, C. Sha, and S. Yu, "Adaptive and extensible energy supply mechanism for UAVs-aided wireless-powered Internet of things," IEEE Internet Things J., vol. 7, no. 9, pp. 9201-9213, Sep. 2020.

[35] S. Nakamura and H. Hashimoto, "Error characteristic of passive position sensing via coupled magnetic resonances assuming simultaneous realization with wireless charging," IEEE Sensors J., vol. 15, no. 7, pp. 3675-3686, Jul. 2015.

[36] Z. Zhang and B. Zhang, "Omnidirectional and efficient wireless power transfer system for logistic robots," IEEE Access, vol. 8, pp. 13683-13693, 2020

[37] H. Haus, Waves and Fields in Optoelectronics. Englewood Cliffs: Prentice-Hall, 1984.

[38] C. J. Chen, T. H. Chu, C. L. Lin, and Z. C. Jou, "A study of loosely coupled coils for wireless power transfer," IEEE Trans. Circuits and Syst. II: Express Briefs, vol. 57, no. 7, pp. 536-540, July 2010.

[39] S. Cheon, Y. H. Kim, S. Y. Kang, M. L. Lee, J. M. Lee, and T. Zyung, "Circuit-model-based analysis of a wireless energy-transfer system via coupled magnetic resonances," IEEE Trans. Ind. Electron., vol. 58, no. 7, pp. 2906-2914, Jul. 2011.

[40] M. Kiani and M. Ghovanloo, "The circuit theory behind coupled-mode magnetic resonance-based wireless power transmission," IEEE Trans. Circuits Syst. I, vol. 59, no. 8, pp. 1-10, Aug. 2012.

[41] D. Y. Lin, C. Zhang, and S. Y. R. Hui, "Mathematical analysis of omnidirectional wireless power transfer-Part-I: Two-dimensional systems," IEEE Trans. Power Electron., vol. 32, no. 1, pp. 625-633, Jan. 2017.

[42] D. Y. Lin, C. Zhang, and S. Y. R. Hui, "Mathematical analysis of omnidirectional wireless power transfer-Part-II: Three-dimensional systems," IEEE Trans. Power Electron., vol. 32, no. 1, pp. 613-624, Jan. 2017.

[43] R. J. Garbacz, "A generalized expansion for radiated and scattered fields," Ph.D. dissertation, Dept. Elect. Eng., The Ohio State Univ., Columbus, OH, USA, 1968.

[44] R. J. Garbacz and R. H. Turpin, "A generalized expansion for radiated and scattered fields," IEEE Trans. Antennas Propag., vol. AP-19, no. 3, pp. 348-358, May 1971.

[45] R. F. Harrington and J. R. Mautz, Theory and Computation of Characteristic Modes for Conducting Bodies. Interaction Notes, Note 195, Syracuse Univ., Syracuse, New York, USA, Dec. 1970.

[46] R. F. Harrington and J. R. Mautz, "Theory of characteristic modes for conducting bodies," IEEE Trans. Antennas Propag., vol. AP-19, no. 5, pp. 622-628, Sep. 1971.

[47] R. Z. Lian, "Research on the work-energy principle based characteristic mode theory for scattering systems," Ph.D. dissertation, School of Electronic Science and Engineering, University of Electronic Science and Technology of China (UESTC), Chengdu, Sichuan, China, 2019. [Online]. Available: https://arxiv.org/abs/1907.11787.

[48] R. Z. Lian, X. Y. Guo, and M. Y. Xia, "Work-energy principle based characteristic mode theory with solution domain compression for material scattering systems," with identifier [AP2004-0708] submitted to IEEE Trans. Antennas Propag., on April 11, 2020.

[49] B. N. Parlett, The Symmetric Eigenvalue Problem. Englewood Cliffs: Prentice-Hall, 1980. 
[50] R. Lian and J. Pan, "Method for constructing the non-radiative characteristic modes of perfect electric conductors," Electron. Lett., vol. 54, no. 5, pp. 261-262, Mar. 2018.
Ren-Zun Lian received the B.S. degree in optical engineering from the University of Electronic Science and Technology of China (UESTC), Chengdu, China, in 2011, and received the $\mathrm{Ph} . \mathrm{D}$. degree in electromagnetic field and microwave technology from UESTC, Chengdu, China, in 2019.

He is currently a postdoctoral researcher in Peking University (PKU), Beijing, China. His current research interests include mathematical physics, electromagnetic theory and computation, and antenna theory and design.

Ming-Yao Xia (M'00-SM'03) received the Master and Ph. D degrees in electrical engineering from the Institute of Electronics, Chinese Academy of Sciences (IECAS), in 1988 and 1999, respectively. From 1988 to 2002, he was with IECAS as an Engineer and a Senior Engineer. He was a Visiting Scholar at the University of Oxford, U.K., from October 1995 to October 1996. From June 1999 to August 2000 and from January 2002 to June 2002, he was a Senior Research Assistant and a Research Fellow, respectively, with the City University of Hong Kong. He joined Peking University as an Associate Professor in 2002 and was promoted to Full Professor in 2004. He moved to the University of Electronic Science and Technology of China as a Chang-Jiang Professor nominated by the Ministry of Education of China in 2010. He returned to Peking University after finishing the appointment in 2013. He was a recipient of the Young Scientist Award of the URSI in 1993. He was awarded the first-class prize on Natural Science by the Chinese Academy of Sciences in 2001. He was the recipient of the Foundation for Outstanding Young Investigators presented by the National Natural Science Foundation of China in 2008. He served as an Associate Editor for the IEEE Transactions on Antennas and Propagation. His research interests include electromagnetic theory, numerical methods and applications, such as wave propagation and scattering, electromagnetic imaging and probing, microwave remote sensing, antennas and microwave components.

Xing-Yue Guo (S'17) received the B.S. degree in electronic information science and technology from the Southwest Jiaotong University, Chengdu, China, in 2011, and the M.E. degree from the University of Electronic Science and Technology of China, Chengdu, China, in 2014. From 2014 to 2017, she was a Research Assistant with the CAEP Software Center for High Performance Numerical Simulation, Beijing, China. Since 2017, she has been pursuing the Ph. D. degree at the School of Electronics Engineering and Computer Science, Peking University, Beijing, China. Her research interests include computational electromagnetics and applications. 